Pacific

Journal of

Mathematics

\title{
INTEGRAL SPECIALIZATION OF FAMILIES OF RATIONAL FUNCTIONS
}

Pierre Dèbes And Michael D. Fried 


\title{
INTEGRAL SPECIALIZATION OF FAMILIES OF RATIONAL FUNCTIONS
}

\author{
Pierre Dèbes and Michael D. Fried
}

\begin{abstract}
Suppose $C$ is an algebraic curve, $f$ is a rational function on $C$ defined over $\mathbb{Q}$, and $\mathcal{A}$ is a fractional ideal of $\mathbb{Q}$. If $f$ is not equivalent to a polynomial, then Siegel's theorem gives a necessary condition for the set $C(\mathbb{Q}) \cap f^{-1}(\mathcal{A})$ to be infinite: $C$ is of genus 0 and the fiber $f^{-1}(\infty)$ consists of two conjugate quadratic real points. We consider a converse. Let $\mathcal{P}$ be a parameter space for a smooth family $\Phi: \mathcal{T} \rightarrow \mathcal{P} \times \mathbb{P}^{\mathbf{1}}$ of (degree $n$ ) genus 0 curves over $\mathbb{Q}$. That is, the fiber $\mathcal{T}_{p}$ of points of $\mathcal{T}$ over $\boldsymbol{p} \times \mathbb{P}^{\mathbf{1}}$ has genus 0 for $\boldsymbol{p} \in \mathcal{P}$. Assume a Zariski dense set of $\boldsymbol{p} \in \mathcal{P}(\mathbb{Q})$ have fiber $\Phi_{\boldsymbol{p}}^{-1}(\boldsymbol{p} \times \infty)$ over $\infty$ consisting of two conjugate quadratic real points. The family $\Phi$ is then a Siegel family. We ask when the conclusion of Siegel's theorem - $\Phi_{p}(\mathbb{Q}) \cap \mathcal{A}$ is infinite - holds for a Zariski dense subset of $\boldsymbol{p} \in \mathcal{P}(\mathbb{Q})$.

We show how braid action on covers and Hurwitz spaces can tackle this. It refines a unirationality criterion for Hurwitz spaces. A particular family, ${ }_{10} \Phi^{\prime}$, of degree 10 rational functions, illustrates this. It arises as the exceptional case for a general result on Hilbert's Irreducibility Theorem. Fried, 1986 says the only indecomposable polynomials $f(y) \in \mathbb{Q}[y]$ with $f(y)-t$ reducible in $\mathbb{Q}[y]$ for infinitely many $t \in \mathcal{A} \backslash f(\mathbb{Q})$ have degree 5 . We show the family ${ }_{10} \Phi^{\prime}$ satisfies the converse to Siegel's theorem. Thus, exceptional polynomials of degree 5 in Fried, 1986 do exist.

We suspect this result generalizes, thus codifying arithmetic accidents occurring in ${ }_{10} \mathcal{P}^{\prime}$. To illustrate, we've cast this paper as a collection of elementary group theory tools for extracting from a family of covers special cases with specific arithmetic properties. Examples of Siegel and Néron families show the efficiency of the tools, though each case leaves a diophantine mystery.
\end{abstract}

\section{Introduction and the basic families.}

This paper completes part of [DF90a]. It is a tools paper, illustrating with one main (and several support) examples how to analyze a certain style of question. When a diophantine or geometric problem about curves 
defines possible exceptional cases, how do you find if the exceptions do exist? In practice the production of illustrative curves occurs with extra data. That data usually includes a reference variable defining the curve as a cover. That is why, in practice, Hurwitz-style families of covers are natural. Our illustrations especially discuss tools for treating the value sets of genus 0 covers. This will show elementary use of Hurwitz families and braid group action as does [Deb99], [Fri90, Fri95b, Fri95a], [MM] and [Völ96]. [Ser92] has simpler examples not requiring Hurwitz families and braid groups.

This paper culminates an era in which the monodromy method has proved itself innumerable times on many problems of renown. It is time to say these (as delineated here) are the elementary tools. The program Modular Towers of [Fri95c] has outlined the next stage of problems. It requires a set of tools (like modular representation theory and higher monodromy) that divides it from the tools here. Still, there are many problems around which require neat analysis for which the tools here suffice. We hope the community will use them.

Any cover, $\varphi: X \rightarrow \mathbb{P}_{z}^{1}$ has an associated group $G$ and attached conjugacy classes $\mathrm{C}_{1}, \ldots, \mathrm{C}_{r}$. Newcomers will find [Fri95b] examples an easy introduction to these ideas. As here, they include families of polynomials in practical applications. Group theory in this paper occurs mostly within the groups $S_{5}$ and $S_{10}$. Reader: We act on the right of the integers with permutation groups. That means, (123) times (234) is (13)(24), not (34)(12). Also, the braid group $B_{r}$ acts on the right of Nielsen classes ( $\S 1.1$ ).

Notations. Let $K$ be a field and $\bar{K}$ an algebraic closure. A rational function $f \in K(y)$ is decomposable over $K$ if $f$ is a functional composition $f_{1}\left(f_{2}(y)\right)$ with $\operatorname{deg}\left(f_{i}\right)>1, f_{i}(y) \in K(y)$ for $i=1,2$. Otherwise it is indecomposable over $K$. Suppose $f \in K[y]$ and $(\operatorname{deg}(f)$, $\operatorname{char}(K))=1$. Then, $f$ is indecomposable over $K$ if and only if it is indecomposable over $\bar{K}$ ([FM69] or [Sch82, p. 14]). For $g \in S_{n}$, write $g=\beta_{1} \cdots \beta_{t}$, a product of disjoint cycles (including those of length 1 ), the ind $(g)=n-t$. Each element in the conjugacy class of $g$ has the same index; refer to it as the index of the conjugacy class. This notation appears in the Riemann-Hurwitz formula (1.1).

Finally, we use fiber products of morphisms $\psi_{i}: Y_{i} \rightarrow X, i=1,2$. Basically, these are pairs $\left\{\left(y_{1}, y_{2}\right) \in Y_{1} \times Y_{2} \mid \psi_{1}\left(y_{1}\right)=\psi_{2}\left(y_{2}\right)\right\}$. Often, however, this set theoretic product has singularities, though $Y_{1}$ and $Y_{2}$ are nonsingular varieties. Example: If $\psi_{1}$ and $\psi_{2}$ are ramified over a common point, the fiber product has a singular point at the pair of ramified points. So, it is usual to normalize the variety to remove some (all for curves) of this singularity. Even after normalizing the fiber product notation is reminiscent of $Y_{1} \times_{X} Y_{2}$.

1.1. An exceptional family of covers represented by polynomials. A family, of genus zero covers (represented by polynomial maps), appears 
in [DF90a, §2.1]. Each cover in this family has $r=4$ branch points. Its parameter space $\mathcal{H}_{\infty}=\mathcal{H}_{\infty}\left(S_{5}, \mathbf{C}\right)$ is a natural closed subvariety of a minimal (absolute) Hurwitz parameter space $\mathcal{H}=\mathcal{H}\left(S_{5}, \mathbf{C}\right)$. The word absolute here means that two covers $\varphi_{1}: Y_{1} \rightarrow \mathbb{P}_{z}^{1}$ and $\varphi_{2}: Y_{2} \rightarrow \mathbb{P}_{z}^{1}$, corresponding to distinct points $\boldsymbol{p}_{1}, \boldsymbol{p}_{2} \in \mathcal{H}$, are inequivalent: There exists no continuous map $\psi: Y_{1} \rightarrow Y_{2}$ for which $\varphi_{2} \circ \psi=\varphi_{1}$.

Any smooth irreducible family of covers with each cover having $r$ (fixed) branch points has an associated group $G$ and a set of conjugacy classes $\mathbf{C}=\left(\mathrm{C}_{1}, \ldots, \mathrm{C}_{r}\right)$ of $G$. In our special case: $G=S_{5}, r=4, \mathrm{C}_{2}=\mathrm{C}_{3}$ is the class of 2-cycles, $\mathrm{C}_{1}$ the class of products of two disjoint 2-cycles and $\mathrm{C}_{4}$ that of 5-cycles. A summary of this is in the data for the associated Nielsen class:

$$
\mathrm{Ni}=\mathrm{Ni}\left(S_{5}, \mathbf{C}\right)=\left\{\boldsymbol{g}=\left(g_{1}, \ldots, g_{4}\right) \mid g_{1} g_{2} g_{3} g_{4}=1,\langle\boldsymbol{g}\rangle=S_{5} \text { and } \boldsymbol{g} \in \mathbf{C}\right\}
$$

The expression $\boldsymbol{g} \in \mathbf{C}$ means these $g_{i} \mathrm{~s}, i=1,2,3,4$, are in the respective conjugacy classes $\mathrm{C}_{i}, i=1,2,3,4$, in some order.

Notice that $S_{5}$ acts on $\operatorname{Ni}\left(S_{5}, \mathbf{C}\right)$ by conjugation: For $g \in S_{5}, \boldsymbol{g} \in \operatorname{Ni}\left(S_{5}, \mathbf{C}\right)$, $\boldsymbol{g} \mapsto g \boldsymbol{g} g^{-1}$. Denote the quotient $\mathrm{Ni} / S_{5}$ by Ni ${ }^{\text {abs }}$. Riemann's Existence Theorem (RET) gives a simple interpretation of the elements of this Nielsen class. Fix the four branch points as $z^{0}=\left(z_{1}^{0}, \ldots, z_{4}^{0}\right)$. RET says elements of $\mathrm{Ni}^{\text {abs }}$ correspond one-one with equivalence classes of covers having branch points $z^{0}$ and associated conjugacy classes and group. We say these covers are in the Nielsen class.

Denote elements $\boldsymbol{g} \in \mathrm{Ni}$ with $g_{4} \in \mathrm{C}_{4}$ by $\mathrm{Ni}_{\infty}$. Let $\varphi: X \rightarrow \mathbb{P}_{z}^{1}$ be a (degree 5) cover in this Nielsen class. This cover canonically determines a fixed branch point in the family corresponding to the conjugacy class $\mathrm{C}_{4}$. It is the unique point $z^{\prime}$ having just one point of $X$ over it. Let $\lambda$ be any linear fractional transformation taking $z^{\prime}$ to $\infty$. The cover $\lambda \circ \varphi: X \rightarrow \mathbb{P}_{z}^{1}$ is in the same Nielsen class and has $\infty$ as its branch point associated to $\mathrm{C}_{4}$. This $\mathrm{SL}_{2}(\mathbb{C})$ action is a special case of an action $\S 6.2$ discusses briefly. In our example family, it normalizes coordinates to use this observation and consider only the sublocus (that is the space $\mathcal{H}_{\infty}$ ) in the complete family $\mathcal{H}$ where a designated fourth branch point is $\infty$.

The reader might wish that we use this normalization and immediately write out coordinates expressing our example as a family of polynomials. This is sensible, reasonable and yet, probably impossible as $\S 1.7$ explains. $\S 2.3$ uses another parameter space $\mathcal{P}_{\alpha, \beta, c, d}$, pullback from $\mathcal{H}$. This has explicit coordinates that we combine with the following property. $\S 1.5$ produces a new family over the parameter space $\mathcal{H}$ (and $\mathcal{H}_{\infty}$ ) whose fibers are degree 10 covers of $\mathbb{P}_{z}^{1}$. Pull this family back over $\mathcal{P}_{\alpha, \beta, c, d}$ and for any rational point in $\mathcal{P}_{\alpha, \beta, c, d}$, the fiber over this point has infinitely many rational points. 
This rational-points-in-fibers property doesn't hold for $\mathcal{H}_{\infty}$. This is so significant that distinctions between these families is a major subtheme of this paper. The $\mathrm{SL}_{2}(\mathbb{C})$ observation above, however, implies the $\mathrm{SL}_{2}(\mathbb{C})$ quotient of $\mathcal{H}$, and an analogous quotient of $\mathcal{H}_{\infty}$ are the same. This turns a crucial task, showing these particular families have only one irreducible $\mathbb{Q}$-component, into group theory calculation. Subsections 1.2 and 1.3 show how the braid group produces this family from the Nielsen class.

1.2. Enter the braid group. Suppose a cover $\varphi: X \rightarrow \mathbb{P}_{z}^{1}$ is in a given Nielsen class. So an $r$-tuple from the Nielsen class is a branch cycle description of the cover. The Riemann-Hurwitz formula gives the covering curve genus $g$ as

$$
2(n+g-1)=\sum_{i=1}^{r} \operatorname{ind} \mathrm{C}_{i}, n=\operatorname{deg}(\varphi) .
$$

For the particular Nielsen class $\mathrm{Ni}\left(S_{5}, \mathbf{C}\right)$ the genus satisfies $2(5+g-1)=$ $2+1+1+4=8$, or $g=0$. Such branch cycle descriptions correspond to polynomial covers (as below).

This example uses $\mathrm{Ni}_{\infty}$ modulo the conjugation action of $S_{5}: \mathrm{Ni}_{\infty}^{\text {abs }}=$ $\mathrm{Ni}_{\infty} / S_{5}$. All classes, modulo $S_{5}$, have a representative with $g_{4}$ equal $g_{\infty}=$ $(12345)^{-1}$. Further normalize by conjugating by a power of $g_{\infty}$. Thus assume the entry that is a product of two disjoint 2-cycles (type (2)(2)) fixes 1. Suppose the $(2)(2)$ type element is in the first position. The following lists the first three elements in these normalized entries of $\mathrm{Ni}_{\infty}$.

List $\mathrm{A} . \mathrm{Ni}_{\infty}^{\mathrm{abs}}$ representatives having $(2)(2)$ in first position
a) $((23)(45),(12),(14))$
b) $((23)(45),(14),(24))$
c) $((23)(45),(24),(12))$
d) $((25)(34),(12),(35))$
e) $((25)(34),(35),(12))$.

The braid group $B_{r}$ has generators $Q_{1}, \ldots, Q_{r-1}$ modulo the two relations

$$
\begin{aligned}
Q_{i} Q_{j} & =Q_{j} Q_{i} \quad \text { for }|i-j|>1 \quad \text { and } \\
Q_{i+1} Q_{i} Q_{i+1} & =Q_{i} Q_{i+1} Q_{i}, \quad 1 \leq i \leq r-2 .
\end{aligned}
$$

There are two other significant words:

(1.3a) $Q_{1} \cdots Q_{r-1} Q_{r-1} \cdots Q_{1} ;$ and

(1.3b) $\left(Q_{1} \cdots Q_{r-1}\right)^{r}$.

The Hurwitz monodromy group $H_{r}$ is the quotient of $B_{r}$ modulo (1.3a). The mapping class group $M_{r}$ is the quotient of $H_{r}$ modulo (1.3b). Most significant, the braid group acts on a Nielsen class. For $\boldsymbol{g} \in \mathrm{Ni}(G, \mathbf{C})$,

$$
\begin{aligned}
(\boldsymbol{g}) Q_{i}=\left(g_{1}, \ldots, g_{i-1}, g_{i} g_{i+1} g_{i}^{-1}, g_{i}, g_{i+2}, \ldots, g_{r}\right) & \\
& i=1, \ldots, r-1 .
\end{aligned}
$$


The word $w \in B_{r}$ appearing in (1.3a) conjugates $\boldsymbol{g}$ by $g_{1}$ :

$$
\boldsymbol{g} \mapsto(\boldsymbol{g}) w=g_{1} \boldsymbol{g} g_{1}^{-1}=\left(\ldots, g_{1} g_{i} g_{1}^{-1}, \ldots\right)
$$

A list similar to List A has the (2)(2) type element in the 2 nd or in the 3rd position. To get these, apply braid elements $Q_{1}$ and $Q_{2} Q_{1}$ to List A.

Riemann's Existence Theorem interprets List A. Polynomials $h_{1}$ and $h_{2}$ are affine equivalent if $h_{1}(a y+b)=h_{2}(y)$ for some $a \in \mathbb{C} \backslash\{0\}, b \in \mathbb{C}$. Given three distinct values, $z_{1}, z_{2}, z_{3} \in \mathbb{C}$, up to affine equivalence there are exactly five polynomials $f$ of degree 5 with these properties.

(1.5a) $f: \mathbb{P}_{y}^{1} \rightarrow \mathbb{P}_{z}^{1}$ has $z_{1}, z_{2}, z_{3}$ as its finite branch points.

(1.5b) The Galois closure of the cover (1.5a) has group $S_{5}$.

(1.5c) Inertia group generators for ramification from the cover by $f$ over the three branch points are in $\mathrm{C}_{1}, \mathrm{C}_{2}$ and $\mathrm{C}_{3}$, in that order.

Thus, there are exactly 15 equivalence classes of polynomials without stipulating the order of attachment of the $z_{i} \mathrm{~s}$ to the conjugacy classes in (1.5c). We say a polynomial, with a description from List A, or its images under $Q_{1}$ or $Q_{1} Q_{2}$, is in the Nielsen class $\operatorname{Ni}\left(S_{5}, \mathbf{C}\right)$.

[DF90a, $\S 1.3]$ illustrates braid group action on elements of a Nielsen class. As above, denote equivalence classes of covers of $\mathbb{P}_{z}^{1}$ in $\mathrm{Ni}_{\infty}$ by $\mathcal{H}_{\infty}$. For each $\boldsymbol{p} \in \mathcal{H}_{\infty}$, there exists $f_{\boldsymbol{p}}: \mathbb{P}^{1} \rightarrow \mathbb{P}^{1}$, a polynomial mapping in the equivalence class of $\boldsymbol{p}$.

1.3. $\mathcal{H}_{\infty}$ and a cover of the $\lambda$-line. Let $\mathbb{A}^{n}$ be affine $n$-space. Denote the fat diagonal of $\mathbb{A}^{n}$ by $\Delta_{n}$. This is the set of coordinates with two or more entries equal. Then, $S_{n}$ also acts on $\mathbb{A}^{n} \backslash \Delta_{n}$; the quotient $\mathbb{A}^{n} \backslash \Delta_{n} / H$ by any subgroup $H$ of $S_{n}$ is an affine variety. Denote the quotient $\mathbb{A}^{3} \backslash \Delta_{3} /\langle(23)\rangle$ by $U_{\infty}$.

Let $f$ be a degree 5 polynomial in $\mathrm{Ni}_{\infty}$. Then, $D(f)=\left(z_{1},\left\{z_{2}, z_{3}\right\}\right)$ signifies the unordered pair $\left\{z_{2}, z_{3}\right\}$ corresponds to the 2-cycle branch points. The $(2)(2)$ type branch point of $f$ is $z_{1}$. Identify points of $U_{\infty}$ with these symbols. Similarly, let $U_{3}$ be the quotient of $\mathbb{A}^{3} \backslash \Delta_{3}$ by $S_{3}$. Let $D^{\prime}(f)$ be the unordered triple $\left\{z_{1}, z_{2}, z_{3}\right\} \in U_{3}$ of branch points of $f$.

This notation applies to any covers in a Nielsen class (no polynomial structure is necessary). Then, $D: \mathcal{H}_{\infty} \rightarrow U_{\infty}$ is a degree 5 unramified covering map and $D^{\prime}: \mathcal{H}_{\infty} \rightarrow U_{3}$ is a degree 15 unramified covering map. Permutation representations of subquotient groups of $B_{4}$ on $\mathrm{Ni}_{\infty}$ produce these covers. [DF90a, p. 115] introduces $H_{3}^{*}$ : the image in $H_{4}$ of the group $Q_{1}$ and $Q_{2}$ generate. Transitivity of $H_{3}^{*}$ on $\mathrm{Ni}_{\infty}$ is equivalent to irreducibility of $\mathcal{H}_{\infty}$ [DF90a, Prop. 1.10]. Squares of the $Q_{i} \mathrm{~s}, i=1,2$, generate a subgroup $\mathrm{SH}_{3}^{*}$ of $\mathrm{H}_{3}^{*}$. 
Consider the fiber product $\mathcal{H}^{\prime}=\mathcal{H}_{\infty} \times_{U_{3}} \mathbb{A}^{3} \backslash \Delta_{3}$ of $\mathcal{H}_{\infty} \rightarrow U_{3}$ and $\mathbb{A}^{3} \backslash \Delta_{3} \rightarrow U_{3}$ (top of $\S 1$ ). This degree 15 cover of $\mathbb{A}^{3} \backslash \Delta_{3}$ has three natural components. One of these is the subspace of $\boldsymbol{p} \in \mathcal{H}^{\prime}$ having this property: If $\boldsymbol{p}$ lies over $\left(z_{1}, z_{2}, z_{3}\right) \in \mathbb{A}^{3} \backslash \Delta_{3}$, then $z_{1}$ is the (2)(2) type branch point of $f_{\boldsymbol{p}}$. Call this space $\mathcal{H}^{\infty}$; it is an irreducible, degree 5 unramified cover of $\mathbb{A}^{3} \backslash \Delta_{3}$, precisely because $S H_{3}^{*}$ is transitive on List A [DF90a, Prop. 1.11]. Denote restriction of $\mathcal{H}^{\infty}$ to the set

$$
\left\{\left(z_{1}, 0,1\right) \mid z_{1} \in \mathbb{P}^{1} \backslash\{0,1, \infty\}\right\}
$$

by $\mathcal{H}_{0,1, \infty}$. (Related calculations might replace 0 by $z_{2}^{0}$ and 1 by $z_{3}^{0}$.) Then, $\mathcal{H}_{0,1, \infty}$ is irreducible precisely because $a_{1,2}$ and $a_{1,3}$ below act transitively on List A [BF82, §1 Lemma 1.6] (see (1.6) and §6). Compatible with the treatment of $\S 6.2$, the variable $z_{1}$ is the traditional variable $\lambda$ from modular functions.

Label elements of $\mathrm{Ni}_{\infty}^{\text {abs }}$ from List $\mathrm{A}$ as $\left\{0_{a}, 0_{b}, 0_{c}, 0_{d}, 0_{e}\right\}$. While $Q_{3}$ doesn't act on $\mathrm{Ni}_{\infty}$, its square and the following elements $a_{1,2}, a_{1,3}$ and $a_{1,4}$ do. Here are specific actions from (1.4):

$$
a_{1,2}=Q_{1}^{-2}=\left(0_{a} 0_{d} 0_{b}\right)\left(0_{c} 0_{e}\right), a_{1,3}=Q_{1} Q_{2}^{-2} Q_{1}^{-1}=\left(0_{a} 0_{c} 0_{e}\right)\left(0_{b} 0_{d}\right) .
$$

These generate $S_{5}$ itself - a transitive group - acting on List A. Here, where $r=4$, consider a 3rd element's action on List A:

$$
a_{1,4}=Q_{1} Q_{2} Q_{3}^{-2} Q_{2}^{-1} Q_{1}^{-1}=\left(0_{c} 0_{b} 0_{a}\right) .
$$

From (1.3a), $a_{1,2} a_{1,3} a_{1,4}=\left(Q_{1} Q_{2} Q_{3}^{2} Q_{2} Q_{1}\right)^{-1}$ acts trivially on $\mathrm{Ni}_{\infty}^{\text {abs }}$. Thus, the actions of $a_{1,2}, a_{1,3}$ and $a_{1,4}$ on List $\mathrm{A}$ give branch cycles for a degree 5 cover $\bar{\varphi}: \overline{\mathcal{H}}_{0,1, \infty} \rightarrow \mathbb{P}_{z_{1}}^{1}$ of the sphere. Three values of $z_{1}$ ramify in $\bar{\varphi}:$ $0,1, \infty$. Formula (1.1) says the genus $g$ of $\overline{\mathcal{H}}_{0,1, \infty}$ is $0: 2(5+g-1)=$ $\operatorname{ind}\left(a_{1,2}\right)+\operatorname{ind}\left(a_{1,3}\right)+\operatorname{ind}\left(a_{1,4}\right)=3+3+2$. For this and future reference, Prop. 6.5 explains (based on [BF82]) how this calculation shows $\mathcal{H}_{\infty}$ has a function field that is a subfield of a pure transcendental field over $\mathbb{Q}$. That is, $\mathcal{H}_{\infty}$ is unirational, and $\mathcal{H}_{\infty}(\mathbb{Q})$ is dense.

[DF90a] also wrote explicit coordinates for $\mathcal{H}_{\infty}$ revealing this directly. Usually, however, such computation is too difficult. The proof of Main Theorem 1.2 does rely on these coordinates. We don't know if it is possible to replace these special manipulations with a straightforward group criterion. Group theory provides more efficient (and intrinsic) interpretation than does the shape of the equations for these parameter spaces and fiber products.

There is a total family of representing covers over the space $\mathcal{H}_{\infty}$. Specifically, there is a smooth, absolutely irreducible, variety $\mathcal{T}_{5}$ with $\Psi_{5}: \mathcal{T}_{5} \rightarrow$ $\mathcal{H}_{\infty} \times \mathbb{P}_{z}^{1}$ a finite degree 5 map having the following properties.

(1.8a) $\Psi_{5}$ and $\mathcal{T}_{5}$ have field of definition $\mathbb{Q}$.

(1.8b) $\mathcal{T}_{5}$ is absolutely irreducible. 
(1.8c) For $\boldsymbol{p} \in \mathcal{H}_{\infty}$, the fiber over $\boldsymbol{p}$ is a cover $\Psi_{5, \boldsymbol{p}}: \mathcal{T}_{5, \boldsymbol{p}} \rightarrow \mathbb{P}_{z}^{1}$ equivalent to a polynomial map $f_{\boldsymbol{p}}: \mathbb{P}_{y}^{1} \rightarrow \mathbb{P}_{z}^{1}$.

Equivalence in (1.8c) means there exists $\alpha: \mathcal{T}_{5, \boldsymbol{p}} \rightarrow \mathbb{P}_{y}^{1}$ with $f_{\boldsymbol{p}} \circ \alpha=\Psi_{5, \boldsymbol{p}}$, restriction of $\Psi_{5}$ to the fiber over $\boldsymbol{p}$.

Existence (and uniqueness) of $\Psi_{5}$ is a consequence of the following property of the absolute Nielsen class. The subgroup $S_{4}$ of $S_{5}$ defines the degree 5 coset representation giving the monodromy group of the cover. Also, $S_{4}$ is self-normalizing - consisting exactly of elements in $S_{5}$ normalizing $S_{4}$ [Fri77, §1]. This means covers in the family have no automorphisms. In particular, $\mathcal{H}_{\infty}$ is an irreducible fine moduli space for equivalence classes of covers in this Nielsen class.

Another group property translates arithmetically (see references above). As all conjugacy classes in $S_{n}$ are rational, $\mathbf{C}$ consists of rational classes of $S_{5}$. This guarantees $\mathcal{H}_{\infty}, \mathcal{H}_{0,1, \infty}$ and the total families over them, have field of definition $\mathbb{Q}[$ Fri77, Thm. 5.1].

1.4. HIT motivation from [Fri86] for (1.8). Let $\mathcal{A}$ be a fractional ideal of $\mathbb{Q}$. For $f \in \mathbb{Q}[y]$ denote $\left\{z_{0} \in \mathcal{A} \mid f(y)-z_{0}\right.$ is reducible $\}$ by $\mathcal{R}_{f}(\mathcal{A})$. Also, denote $\mathcal{A} \cap f(\mathbb{Q})$ by $\mathcal{V}_{f}(\mathcal{A})$. Use the notation $f \in S_{\mathcal{A}}$ if the following holds:

(1.9) $f \in \mathbb{Q}[y]$ with $\mathcal{R}_{f}(\mathcal{A}) \backslash \mathcal{V}_{f}(\mathcal{A})$ infinite.

Theorem 1.1 (Explicit HIT Theorem [Fri86]). Suppose $f \in S_{\mathcal{A}}$. Then:

(1.10a) Either $\operatorname{deg}(f)=5$; or

(1.10b) $f$ is decomposable over $\mathbb{Q}$ (top of $\S 1$ ).

Further, if (1.10b) doesn't hold, then either $f$ is in the family (1.8c), or coalescing from (1.8) gives $f$ as a cover with only two finite branch points $(\S 3.3)$.

[DF90a] discussed geometric and arithmetic properties of family (1.8). What it didn't show is that family (1.8) genuinely gives nontrivial examples of $f \in S_{\mathcal{A}}$. For $\boldsymbol{p} \in \mathcal{H}_{\infty}(\mathbb{Q})$, Main Theorem 1.2 explains when the corresponding polynomial cover $f_{\boldsymbol{p}}: \mathbb{P}_{y}^{1} \rightarrow \mathbb{P}_{z}^{1}$ is in $S_{\mathcal{A}}$. An auxiliary cover $h_{f}: \mathbb{P}_{w}^{1} \rightarrow \mathbb{P}_{z}^{1}$ plays a role in that result.

Form the 2-times fiber product of $f_{\boldsymbol{p}}$. Natural notation for this is

$$
Z_{\boldsymbol{p}}=\mathbb{P}_{y}^{1} \times_{\mathbb{P}_{z}^{1}} \mathbb{P}_{y}^{1}=\left\{\left(y_{1}, y_{2}\right) \mid f_{\boldsymbol{p}}\left(y_{1}\right)=f_{\boldsymbol{p}}\left(y_{2}\right)\right\} .
$$

This contains the diagonal component, $\Delta_{2}=\left\{(y, y) \mid y \in \mathbb{P}^{1}\right\}$. Remove $\Delta_{2}$ from $Z_{\boldsymbol{p}}$. The group $S_{2}$ acts on what remains by mapping $\left(y_{1}, y_{2}\right)$ to $\left(y_{2}, y_{1}\right)$. Form the quotient by $S_{2}$. Then normalize what you get in its function field. This gives a projective nonsingular degree 10 cover $\Psi_{5, \boldsymbol{p}}^{(2)}: \mathcal{T}_{5, \boldsymbol{p}}^{(2)} \rightarrow \mathbb{P}_{z}^{1}$. Symmetric functions in $\left(y_{1}, y_{2}\right)$, subject to $f_{\boldsymbol{p}}\left(y_{1}\right)=f_{\boldsymbol{p}}\left(y_{2}\right)$, generate the function field of $\mathcal{T}_{5, \boldsymbol{p}}^{(2)}$. 
1.5. Properties of $\Psi_{5, \boldsymbol{p}}^{(2)}$. Unordered pairs (ten) of distinct integers from $\{1, \ldots, 5\}$ are letters for a permutation representation $R: S_{5} \rightarrow S_{10}$. The cover $\Psi_{5, p}^{(2)}$ has geometric monodromy group $S_{5}$ as a subgroup of $S_{10}$ through this representation. Let $\boldsymbol{g}$ be a branch cycle description of the cover $f_{\boldsymbol{p}}$. Riemann-Hurwitz gives the genus $g$ of $\mathcal{T}_{5, \boldsymbol{p}}^{(2)}$ [DF90a, p. 109]:

$$
2(10+g-1)=\sum_{i=1}^{4} \operatorname{ind}\left(R\left(g_{i}\right)\right) .
$$

If $g_{1}$ and $g_{2}$ are 2-cycles from $S_{5}$, then $R\left(g_{i}\right)$ has shape $(2)(2)(2), i=1,2$. Similarly, if $g_{3}$ is the product of two disjoint 2-cycles, then $R\left(g_{3}\right)$ has shape $(2)(2)(2)(2)$. Finally, $R\left(g_{4}\right)$ has shape $(5)(5)$. Thus, the total contribution to the right side of (1.11) is $2 \cdot 3+4+2 \cdot 4=18: g=0$.

Apply the fiber product construction above directly to $\Psi_{5}: \mathcal{T}_{5} \rightarrow \mathcal{H}_{\infty} \times \mathbb{P}_{z}^{1}$. As above, quotient by $S_{2}$ and normalize to get

$$
\Psi_{5}^{(2)}: \mathcal{T}_{5}^{(2)} \rightarrow \mathcal{H}_{\infty} \times \mathbb{P}_{z}^{1} .
$$

1.6. Explanation of the Main Theorem. Suppose a polynomial function $f=f_{\boldsymbol{p}}$ corresponds to a point $\boldsymbol{p} \in \mathcal{H}_{\infty}(\mathbb{Q})$. There are two possibilities.

(1.12a) $\mathcal{T}_{5, \boldsymbol{p}}^{(2)}$ has a $\mathbb{Q}$ point and is $\mathbb{Q}$ isomorphic to $\mathbb{P}_{w}^{1}$.

$(1.12 \mathrm{~b}) \mathcal{T}_{5, \boldsymbol{p}}^{(2)}$ has no $\mathbb{Q}$ point and is $\mathbb{Q}$ isomorphic to a conic $C_{\boldsymbol{p}} \subset \mathbb{P}^{2}$.

For case (1.12a), $\Psi_{5, \boldsymbol{p}}^{(2)}: \mathcal{T}_{5, \boldsymbol{p}}^{(2)} \rightarrow \mathbb{P}_{z}^{1}$ is equivalent to a cover $h_{f}: \mathbb{P}_{w}^{1} \rightarrow \mathbb{P}_{z}^{1}$.

Let $\mathcal{A}$ be any nontrivial fractional ideal of $\mathbb{Q}$ and consider $S_{\mathcal{A}}$ from $\S 1.4$. Part I of Th. 1.2 below clarifies the necessary condition for $f \in S_{\mathcal{A}}$ of Th. 1.1. Part II is preliminary to Part III, which is the main motivation of this paper; there are nontrivial examples of $f \in S_{\mathcal{A}}$.

Theorem 1.2 (Main Theorem). I. Suppose $f \in S_{\mathcal{A}}$ is indecomposable ((1.10b) does not hold). Then:

(1.13a) Either $f=f_{\boldsymbol{p}}$ for some $\boldsymbol{p} \in \mathcal{H}_{\infty}(\mathbb{Q})$ and (1.12a) holds; or

(1.13b) $f(y)=\beta(v(\alpha(y)))$ with $v$ among an explicit finite set of polynomials, $\alpha(y)=a y+b$ and $\beta(y)=c y+d$ for some $a, b, c, d \in \mathbb{Q}$.

II. Suppose $(1.13 \mathrm{a})$ holds. Then, $\mathcal{R}_{f}(\mathcal{A})$ is the union of $\left(f(\mathbb{Q}) \cup h_{f}(\mathbb{Q})\right) \cap \mathcal{A}$ with a finite set $V$ and $f(\mathbb{Q}) \cap h_{f}(\mathbb{Q}) \cap \mathcal{A}$ is finite.

III. For each $\mathcal{A},\left\{\boldsymbol{p} \in \mathcal{H}_{\infty} \mid f_{\boldsymbol{p}} \in S_{\mathcal{A}}\right\}$ is Zariski dense.

Much of Parts I and II are in [Fri86] ([Fri99, §1.4] documents most of this). What remains of Part I is to codify the second possibility of Th. 1.1. That is, to show the entire contribution to (1.13b) comes from coalescing branch points of covers in (1.8). $§ 3.2$ shows there are three polynomials $v_{1}, v_{2}, v_{3}$ giving all the covers to which $(1.13 \mathrm{~b})$ refers. $\S 3.3$ explains how each $v_{i}$ derives from coalescing (specializing branch points of) a general 
member of the family over $\mathcal{H}_{\infty}$. For Part II, that leaves only finiteness of $f(\mathbb{Q}) \cap h_{f}(\mathbb{Q}) \cap \mathcal{A}$. This is condition (1.14c) of Prop. 1.3. Part III follows from Part II and the two other assertions of Prop. 1.3. $\S 2.3$ starts the proof of Prop. 1.3. This completes applying [DF90a] to Main Theorem 1.2.

Proposition 1.3 (Main Proposition). In the notation above, the following hold.

$(1.14 \mathrm{a}) \boldsymbol{p} \in \mathcal{H}_{\infty}(\mathbb{Q})$ with $(1.12 \mathrm{a})$ holding are dense.

$(1.14 \mathrm{~b})$ For a dense set of $\boldsymbol{p}$ from $(1.14 \mathrm{a}), h_{f_{\boldsymbol{p}}}(\mathbb{Q}) \cap \mathcal{A}$ is infinite.

$(1.14 \mathrm{c})$ For all $\boldsymbol{p} \in \mathcal{H}_{\infty}(\mathbb{Q}), f_{\boldsymbol{p}}(\mathbb{Q}) \cap h_{f_{\boldsymbol{p}}}(\mathbb{Q}) \cap \mathcal{A}$ is finite.

1.7. Comments on families of rational functions. [DF90a] took a pullback of the family of (1.8) to another space $\mathcal{P}$. This pullback gave simple coordinates for $\mathcal{T}_{5, \boldsymbol{P}}^{(2)}$. $\S 2.1$ includes explicit explanation of this family. It is a family of polynomials. This is more than equivalence of each cover in the family to a polynomial cover.

Definition 1.4. Family of rational functions. Call $\Psi: \mathcal{T} \rightarrow \mathcal{H} \times \mathbb{P}_{z}^{1}$ a smooth family of genus zero covers of degree $n$ if the following hold.

(1.15a) $\Psi$ is a finite morphism of smooth varieties.

(1.15b) For $\boldsymbol{p} \in \mathcal{H}, \mathcal{T}_{\boldsymbol{p}} \rightarrow \mathbb{P}_{z}^{1}$ is a degree $n$ cover of (nonsingular) genus 0 curves.

Call $\Psi$ a family of rational functions if the following hold. There is an isomorphism $\Gamma: \mathcal{T} \rightarrow \mathcal{H} \times \mathbb{P}_{y}^{1}$ and a degree $n$ smooth morphism $\Psi^{\prime}: \mathcal{H} \times \mathbb{P}_{y}^{1} \rightarrow$ $\mathcal{H} \times \mathbb{P}_{z}^{1}$ with $\Psi^{\prime} \circ \Gamma=\Psi$. It is a family of polynomials if the only points in $\mathcal{H} \times \mathbb{P}_{y}^{1}$ over $\mathcal{H} \times \infty \subset \mathcal{H} \times \mathbb{P}_{z}^{1}$ are in $\mathcal{H} \times \infty$.

Recall the component $\mathcal{H}^{\infty}$ of the fiber product $\mathcal{H}_{\infty} \rightarrow U_{3}$ with $\mathbb{A}^{3} \backslash \Delta_{3} \rightarrow$ $U_{3}$ (§1.3). Projection from this component induces a map

$$
H_{\text {et }}^{1}\left(\mathcal{H}_{\infty}, \mathbb{Z} / 2\right) \rightarrow H_{\text {et }}^{1}\left(\mathcal{H}^{\infty}, \mathbb{Z} / 2\right) .
$$

[DF90a, §2.3] produced $\alpha \in H_{\text {et }}^{1}\left(\mathcal{H}_{\infty}, \mathbb{Z} / 2\right)$ with these properties.

(1.16a) $\alpha$ is trivial if and only if $\Psi_{5}: \mathcal{T}_{5} \rightarrow \mathcal{H}_{\infty} \times \mathbb{P}_{z}^{1}$ is a family of rational (or polynomial) functions.

(1.16b) The image $\alpha^{*} \in H_{\text {et }}^{1}\left(\mathcal{H}^{\infty}, \mathbb{Z} / 2\right)$ of $\alpha$ is trivial.

Fibers in a family of rational functions over a $\mathbb{Q}$ point must have many $\mathbb{Q}$ points. [DF90a, Th. 4.2] shows there is a dense set of $\boldsymbol{p} \in \mathcal{H}_{\infty}(\mathbb{Q})$ with no rational point on the fiber $\mathcal{T}_{5, \boldsymbol{p}}^{(2)}$ of the family

$$
\Psi_{5}^{(2)}: \mathcal{T}_{5}^{(2)} \rightarrow \mathcal{H}_{\infty} \times \mathbb{P}_{z}^{1} .
$$

In particular, (1.17) can't be a family of rational functions. Yet, the general $\mathbb{Q}$ fiber of the pullback of (1.17) over $\mathcal{H}^{\infty}$ (see $\S 2.1$ ) does have rational points. 
Problem 1.5. Is $\alpha$ nontrivial (so (1.16a) is not a family of rational functions)? Is the pullback of (1.17) over $\mathcal{H}^{\infty}$ a family of rational functions?

$\S 4.1$ describes other Siegel families appropriate for versions of Main Theorem 1.2. $§ 5.1$ updates Hilbert-Siegel problems analogous to HIT Theorem 1.1. It also discusses Müller's result [Mül96] extending both Theorem 1.1 and results from [Fri86] on varying coefficients of polynomial functions other than the constant term.

\section{Pullback family and proof of Main Theorem.}

[DF90a, §2.1] considers (1.17). This is a family of degree 10 covers - by genus 0 curves - with associated Nielsen class $\mathrm{Ni}\left(S_{5}, \mathbf{C}, R\right)$. Here $R$ indicates the permutation representation of $S_{5}$ in $S_{10}$ is that of the monodromy group of these degree 10 covers. The point $z_{4}=\infty$ ramifies in any fiber $\mathcal{T}_{5, \boldsymbol{p}}^{(2)} \rightarrow \mathbb{P}_{z}^{1}$ with cycle type (5)(5). The first step to prove the Main Theorem is finding a Zariski dense subset of $\boldsymbol{p} \in \mathcal{H}_{\infty}(\mathbb{Q})$ where $\mathcal{T}_{5, \boldsymbol{p}}^{(2)}$ has one (and therefore, infinitely many) $\mathbb{Q}$ points.

2.1. Family from adjoining branch points. Suppose $\mathcal{P}$ is any $\mathbb{Q}$ variety whose $\mathbb{Q}$ points are Zariski dense. If $\beta: \mathcal{P} \rightarrow \mathcal{H}_{\infty}$ is a dominant $\mathbb{Q}$ birational morphism, then any dense set of $\mathbb{Q}$ points in $\mathcal{P}$ will have Zariski dense image in $\mathcal{H}_{\infty}$. Thus, to find Zariski dense $\mathbb{Q}$ points on $\mathcal{H}_{\infty}$, it may pay to pull back to any such variety $\mathcal{P}$. Our choice: Take $\mathcal{P}=\mathcal{H}^{\infty}$ (§1.3). One valuable ingredient is that $\S 6.1$ applied to $\S 1.3$ shows $\mathcal{H}^{\infty}$ is a $\mathbb{Q}$ rational variety.

Denote the pullback of $\mathcal{T}_{5}^{(2)}$ to $\mathcal{H}^{\infty}$ by $\overline{\mathcal{T}}_{5}^{(2)}$. It has the following geometric property [DF90a, §2.1].

(2.1) The natural projection $\bar{\Psi}_{5}^{(2)}: \overline{\mathcal{T}}_{5}^{(2)} \rightarrow \mathcal{H}^{\infty} \times \mathbb{P}_{z}^{1}$ is a family (smooth and irreducible) of degree 10 covers with fibers in $\mathrm{Ni}\left(S_{5}, \mathbf{C}, R\right)$.

Further, the family (2.1) has these crucial arithmetic properties.

(2.2a) Over a Zariski open subset of $\mathcal{H}^{\infty}$, $\operatorname{proj}_{1} \circ \bar{\Psi}_{5}^{(2)}: \overline{\mathcal{T}}_{5}^{(2)} \rightarrow \mathcal{H}^{\infty}$ has a section.

$(2.2 \mathrm{~b})$ For each $\boldsymbol{p}_{0} \in \mathcal{H}^{\infty}(\mathbb{Q}), \overline{\mathcal{T}}_{5, \boldsymbol{p}_{0}}^{(2)}(\mathbb{Q})$ is nonempty.

Here is how you get the section $s$ in $(2.2 \mathrm{a})$.

As in $\S 1.3, z_{2}$ is a branch point of the cover corresponding to the shape (2) conjugacy class in $S_{5}$. In the representation $R$, the conjugacy class has shape $(2)(2)(2)(\S 1.5)$. Let $D_{\boldsymbol{p}}$ be the divisor on $\overline{\mathcal{T}}_{5, \boldsymbol{p}}^{(2)}$ consisting of the three ramified points lying over $z_{2}$ in the map $\bar{\Psi}_{5, \boldsymbol{p}}^{(2)}: \overline{\mathcal{T}}_{5, \boldsymbol{p}}^{(2)} \rightarrow \mathbb{P}_{z}^{1}$. Then, let $D_{\boldsymbol{p}}^{\prime}$ be the divisor of the differential of the function $\bar{\Psi}_{5, \boldsymbol{p}}^{(2)}$. Since $\overline{\mathcal{T}}_{5, \boldsymbol{p}}^{(2)}$ has genus $0, D_{\boldsymbol{p}}^{\prime}$ has degree -2 . On a genus zero curve, all divisors of a given degree are linearly equivalent. Further, on any (nonsingular projective) curve over $K$, functions over $K$ generate any linear system whose divisor has field of 
definition $K$. Thus, the linear equivalence class of $D_{\boldsymbol{p}}+D_{\boldsymbol{p}}^{\prime}$ contains a positive (degree 1) $\mathbb{Q}(\boldsymbol{p})$ divisor. This is therefore a point $s(\boldsymbol{p})$ on $\overline{\mathcal{T}}_{5, \boldsymbol{p}}^{(2)}$. Apply this to a generic point $\boldsymbol{p}$ of $\mathcal{H}^{\infty}$. This defines a map $\boldsymbol{p}_{0} \mapsto s\left(\boldsymbol{p}_{0}\right)$ for $\boldsymbol{p}_{0}$ in a Zariski open subset of $\mathcal{H}^{\infty}$ [DF90a, Ex. 3.5]. The argument applies to each point of $\mathcal{H}^{\infty}(\mathbb{Q})$ providing a rational point on the fiber of that point. Note: $s(\boldsymbol{p})$ is not unique. This gives $(2.2 \mathrm{~b})$.

2.2. Real conjugate points over $\infty$. Consider $\boldsymbol{p} \in \mathcal{H}^{\infty}$. Let $\boldsymbol{z}$ be the image of $\boldsymbol{p}$ in $U_{3}$ as in $\S 2.1$. Consider the behavior over $\infty$ of the cover

$$
\bar{\Psi}_{5, \boldsymbol{p}}^{(2)}: \overline{\mathcal{T}}_{5, \boldsymbol{p}}^{(2)} \rightarrow \mathbb{P}_{z}^{1}
$$

Two points, $x_{1, \infty}, x_{2, \infty}$, on $\overline{\mathcal{T}}_{5, \boldsymbol{p}}^{(2)}$ lie over $z=\infty$. These correspond to the two disjoint 5-cycles of $R\left(g_{4}\right)$. (Recall: $g_{4}$ is the last member of a branch cycle description of the cover.) [DF90b] gave a general test for points in the fiber of a cover $\psi: X \rightarrow \mathbb{P}_{z}^{1}$ to be real. Suppose $\mathbb{R}$ contains a field of definition of $(X, \psi)$. Then, the branch points are permuted by complex conjugation. That is, as a set they are in $\mathbb{R}$. The most interesting case is when the fiber $X_{z_{0}}$ is that over a branch point $z_{0}$. Our explicit description of this family allows checking this less abstractly. Still, the efficient group theoretic approach works on examples like those of $\S 4.2$ (specifically see $\S 4.3 .1$ and $\S 4.3 .2)$ without having their explicit equations.

For $G \leq S_{n}$ denote the normalizer of $G$ in $S_{n}$ by $N_{S_{n}}(G)$. In the general case, a given branch point $z_{i}$ is either in $\mathbb{R}$, or it is complex conjugate to another branch point $z_{i^{\prime}}$. Let $\overline{\mathrm{C}}_{i}$ be the conjugacy class in the group $N_{S_{n}}(G)$ containing $\mathrm{C}_{i}$. If $z_{i}$ and $z_{i^{\prime}}$ are conjugate, then the branch cycle argument of, say, [Fri95a] gives an involution in $N_{S_{n}}(G)$ conjugating $\overline{\mathrm{C}}_{i}$ to $\overline{\mathrm{C}}_{i^{\prime}}$. For $\boldsymbol{p} \in \mathcal{H}_{\infty}(\mathbb{R})$, it is possible $z_{2}$ and $z_{3}$ are complex conjugate. A cover, however, associated to $\boldsymbol{p} \in \mathcal{H}^{\infty}(\mathbb{R})$ must have real branch points. Since our covers will come from points of $\boldsymbol{p} \in \mathcal{H}^{\infty}(\mathbb{R})$, the next lemma suffices. (There is, however, a version for the case where pairs of branch points are complex conjugate.) It illustrates how [Fri95c, App.C] detects real points on Hurwitz spaces.

Lemma 2.1 (Real Point Lemma [DF90b]). Let $z_{1}<z_{2} \cdots<z_{r-1} \in \mathbb{A}^{1}$ be real points. Suppose $\mathbf{C}=\left(\mathrm{C}_{1}, \ldots, \mathrm{C}_{r}\right)$ are conjugacy classes of $G$; classes $\mathrm{C}_{1}, \ldots, \mathrm{C}_{r-1}$ correspond to the branch points $z_{1}, \ldots, z_{r-1}$. Let $\varphi_{i}: X_{i} \rightarrow$ $\mathbb{P}^{1}, i=1, \ldots, t$, be a complete set of representatives of equivalence classes of covers in the absolute Nielsen class $\operatorname{Ni}(G, \mathbf{C})_{\infty}^{\text {abs }}$ having $z_{1}<z_{2} \cdots<$ $z_{r-1}$ and $\infty$ (corresponding to $\mathrm{C}_{r}$ ) as branch points. Let $M$ be those from $\left\{\varphi_{i}: X_{i} \rightarrow \mathbb{P}^{1}\right\}_{i=1}^{t}$ equivalent to a cover with field of definition $\mathbb{R}$. There is a one-one correspondence between elements of $M$ and $\boldsymbol{g}=\left(g_{1}, \ldots, g_{r}\right) \in$ $\mathrm{Ni}(G, \mathbf{C})_{\infty}^{\text {abs }}$ with the following property. There is an involution $C \in N_{S_{n}}(G)$ with

$$
C g_{i} C^{-1}=g_{1} \cdots g_{i-1} g_{i}^{-1} g_{i-1}^{-1} \cdots g_{1}^{-1}, i=1, \ldots, r .
$$


If no elements of $S_{n}$ centralize $G$, then $C=C_{g}$ is unique. Call $C$ the complex conjugation operator associated to a cover with branch cycles $\boldsymbol{g}$ (see statement on the association below).

Let $g_{r}$ be the branch cycle corresponding to $\infty$; assume it nontrivial. Since $g_{1} \cdots g_{r}=1$, this says $C g_{r} C=g_{r}^{-1}$. In particular, $C=C_{\boldsymbol{g}}$ conjugates any disjoint cycle $\beta$ of $g_{r}$ to the inverse of another disjoint cycle in $g_{r}$. If there are exactly two such disjoint cycles, then either $C \beta C=\beta^{-1}$, or $C \beta C \neq$ $\beta^{-1}$. Suppose $\varphi^{\prime}: X^{\prime} \rightarrow \mathbb{P}^{1}$ is a cover from $M$. Let $C^{\prime}$ be the complex conjugation operator corresponding to it by hypothesis. Then, real points of $X^{\prime}$ over $\infty$ correspond exactly to disjoint cycles $\beta$ with $C^{\prime} \beta C^{\prime}=\beta^{-1}$.

The Real Point Lemma 2.1 correspondence comes from a specific bouquet of paths on the punctured sphere $\mathbb{P}^{1} \backslash\{\boldsymbol{z}\}$ [DF90b, §2]. Compute branch cycles of $\varphi_{i}: X_{i} \rightarrow \mathbb{P}^{1}, i=1, \ldots, t$, in Lemma 2.1 from this set of paths to get the correspondence in one direction. Cor. 2.2 requires letters for the representation $R$ :

$$
\begin{aligned}
& s_{i}^{\prime}=\{1, i+1\}, i=1,2,3,4, \quad s_{4+i}^{\prime}=\{2, i+2\}, i=1,2,3, \\
& s_{8}^{\prime}=\{3,4\}, \quad s_{9}^{\prime}=\{3,5\}, \quad s_{10}^{\prime}=\{4,5\} .
\end{aligned}
$$

If $\boldsymbol{p} \in \mathcal{H}^{\infty}(\mathbb{R})$, Cor. 2.2 shows the cover $\left(2.3_{\boldsymbol{p}}\right)$ passes the necessary Siegel Theorem condition for a positive conclusion to Main Proposition 1.3: (1.14b) can't hold unless the two points of $h_{\boldsymbol{p}}^{-1}(\infty)$ are real conjugates.

Corollary 2.2. Consider the fifteen equivalence classes of covers having branch cycles $\boldsymbol{g}$ in $\mathrm{Ni}\left(S_{5}, \mathbf{C}, R\right)$ and three finite branch points $\left(z_{1}, z_{2}, z_{3}\right)=$ $z \in \mathbb{Q}^{3}$. From those five with $g_{1} \in \mathrm{C}_{1}$ (and $g_{4} \in \mathrm{C}_{4}$ ), exactly two have field of definition $\mathbb{R}$. Suppose one of these, $\varphi: X \rightarrow \mathbb{P}^{1}$, has field of definition $\mathbb{Q}$. Let $x_{1, \infty}$ and $x_{2, \infty}$ be the geometric points of $\varphi^{-1}(\infty)$. These are conjugate over $\mathbb{Q}(\sqrt{5})$.

Proof. Apply representation $R$ to elements in List A to get branch cycles for the covers of the corollary (see Lemma 2.1). [DF90a, Lemma 2.1] shows $S_{5}$ is self-normalizing in $S_{10}$. The information that some $\alpha \in S_{5}$ takes $g_{1}$ (product of two disjoint 2-cycles) to $g_{1}^{-1}$ and $g_{4}$ (5-cycle) to its inverse determines $C=R(\alpha)$. For example, apply $R$ to entry c), ((23)(45), (24), (12)) from List A. A calculation shows $C=R((25)(34))$ satisfies the criterion of Lemma 2.1:

$$
C R((24)) C=R((23)(45)(24)(23)(45))=R((35)) .
$$

Also, if some $C$ works for List $\mathrm{A}$ items a) and b), it must be $R(\alpha)$. Note, however,

$$
\begin{aligned}
& C R((12)) C \neq R((23)(45)(12)(23)(45))=R((13)) \quad \text { and } \\
& C R((14)) C \neq R((23)(45)(14)(23)(45))=R((15)) .
\end{aligned}
$$


Similarly, for List A items d) and e): $\alpha$ works for e); nothing works for d).

Now check the Lemma 2.1 criterion for the field of definition of the points over $\infty$ for the covers corresponding to c) and e). Use $i$ in place of $s_{i}^{\prime}$ to simplify notation. The two disjoint cycles of $R\left(g_{4}\right)$ are then $\beta_{1}=(158104)^{-1}$ and $\beta_{2}=(26937)^{-1}$. Apply Lemma 2.1. That our choice of $C$ works and is unique is easy. Now check that conjugation by $C$ takes $\beta_{1}$ to $(851410)^{-1}=$ $\beta_{1}^{-1}$. This shows $x_{1, \infty}$ and $x_{2, \infty}$ have coordinates in $\mathbb{R}$.

Finally, suppose one of these covers, $\varphi: X \rightarrow \mathbb{P}_{z}^{1}$, has field of definition $\mathbb{Q}$. We show $x_{1}=x_{1, \infty}$ and $x_{2}=x_{2, \infty}$ aren't $\mathbb{Q}$ points. Let $\zeta_{5}=e^{\frac{2 \pi i}{5}}$. Consider $f: \mathbb{P}_{y}^{1} \rightarrow \mathbb{P}_{z}^{1}$, the corresponding degree 5 polynomial cover. It totally ramifies over $\infty$. So, all Puiseux expansions for local functions on $\mathbb{P}_{y}^{1}$ over $z=\infty$ embed in $\mathbb{Q}\left(\zeta_{5}\right)\left\{\left\{z^{-1 / 5}\right\}\right\}$ : Laurent series in $z^{-1 / 5}$ over $\mathbb{Q}\left(\zeta_{5}\right)$. Thus, the Galois closure $\Omega$ of the extension $\mathbb{Q}(y) / \mathbb{Q}(z)$ embeds in $\mathbb{Q}\left(\zeta_{5}\right)\left\{\left\{z^{-1 / 5}\right\}\right\}$. Since the Galois closure of the field $\mathbb{Q}(X) / \mathbb{Q}(z)$ is also $\Omega, K=G\left(\mathbb{Q}\left(\zeta_{5}\right) / \mathbb{Q}\right)$ acts on coefficients of Puiseux expansions around $x_{1}$ and $x_{2}$. A generator $\gamma$ of $K$ acting on these expansions has order four. The square of $R(\gamma)$ gives the effect of $C$. So, $\gamma=(2354)$ or its inverse. Conjugation by $R(\gamma)=(1243)(78)(59106)$ takes $\beta_{1}$ to $\left(\beta_{2}\right)^{-2}: x_{1}$ and $x_{2}$ are conjugate over the real quadratic subfield of $\mathbb{Q}\left(\zeta_{5}\right)$.

Remark 2.3. $Q_{1}$ and $Q_{1} Q_{2}$ applied to List $A$. For given $z_{1}, z_{2}, z_{3}$, as in Cor. 2.2, apply $Q_{1}$ and $Q_{1} Q_{2}$ to List A to get 10 other covers in the Nielsen class with the same branch points. Some of these covers will also have field of definition $\mathbb{R}$ and some won't. For example, apply $Q_{1}$ to a) of List A to get $((13),(23)(45),(14))$. This has $C=R((13)(45))$ as complex conjugation operator.

2.3. Proof of Main Prop. 1.3. We must prove (1.14a-c). We follow these points for the rest of $\S 2$.

$\left(\mathbf{P}_{1}\right)$ : For $\boldsymbol{p} \in \mathcal{H}^{\infty}(\mathbb{Q})$, apply [DF90a, $\left.\S 4.4\right]$ to identify $\overline{\mathcal{T}}_{5, \boldsymbol{p}}^{(2)}$ with a conic $\mathcal{C}_{p}$.

$\left(\mathbf{P}_{2}\right)$ : Find a rational point on $\mathcal{C}_{\boldsymbol{p}}$.

$\left(\mathbf{P}_{3}\right)$ : Use Euler's parametrization to identify $\overline{\mathcal{T}}_{5, \boldsymbol{p}}^{(2)}$ with $\mathbb{P}_{w}^{1}$.

$\left(\mathbf{P}_{4}\right)$ : Present the covering map $\bar{\Psi}_{5, \boldsymbol{p}}^{(2)}: \overline{\mathcal{T}}_{5, \boldsymbol{p}}^{(2)} \rightarrow \mathbb{P}_{z}^{1}$ as a function $h_{\boldsymbol{p}}(w)$ in $w$.

$\left(\mathbf{P}_{5}\right)$ : Study values of $h_{\boldsymbol{p}}$.

A further pullback of family (2.1) seems necessary for task $\left(\mathrm{P}_{4}\right)$ for (as in $\S 1.7)$ we need to replace (2.1) by a family of rational functions. [DF90a, Theorem 4.2] (see $\S 2.4$ ) gives $\overline{\mathcal{T}}_{5, \boldsymbol{p}}^{(2)}$ as a cover of $\mathbb{P}_{z}^{1}$ by a conic:

$$
\left\{(U, V) \mid U^{2}+V^{2}-3 U V-\frac{5}{4}(c+d) U+\frac{5}{2}(c+d) V-5 c d=0\right\} .
$$


For later, refer to the parameter space of this family by the notation $\mathcal{P}_{\alpha, \beta, c, d}$. Its points correspond to the parameters $(\alpha, \beta, c, d)$ that appear below. Here $c$ and $d$ are in $\mathbb{Q}$. They are coordinates for the unique ramified points on $\mathcal{T}_{5, \boldsymbol{p}}$ above $z_{2}$ and $z_{3}$, the 2 -cycle branch points. [DF90a, Prop. 2.5] pulls back the family of $(1.8)$ over $\mathbb{G} \times \mathbb{A}^{2} \times \mathbb{G} \stackrel{\text { def }}{=} \mathcal{P}$ with $\mathbb{G}=\mathbb{A}^{1} \backslash\{0\}$. This gives

$$
f_{\alpha, \beta, s, t}(y)=\alpha\left(y^{5} / 5-s y^{4} / 4+2 t y^{3}-5 s t y^{2} / 2+5 t^{2} y\right)+\beta \text {. }
$$

Here $s=c+d, t=c d$. With it is associated an allied parameter space $\mathcal{P}_{\alpha, \beta, s, t}^{\prime}=\mathcal{P}^{\prime}$. Later we use the map $\varphi^{*}: \mathcal{P} \rightarrow \mathcal{H}_{\infty}$ sending $(\alpha, \beta, c, d)$ to the equivalence class of the cover $f_{\alpha, \beta, s, t}: \mathbb{P}_{y}^{1} \rightarrow \mathbb{P}_{z}^{1}$. Apply the fiber product construction of $\S 1.4$ directly to $f_{\alpha, \beta, s, t}$ to produce the pullback of (2.4) to $\mathcal{P}$. Denote this by

$$
\bar{\Psi}_{\alpha, \beta, s, t}: \overline{\mathcal{C}}_{\alpha, \beta, s, t} \rightarrow \mathbb{P}_{z}^{1}
$$

as in [DF90a, §4.4]. Equation (2.4) is an affine portion of (2.5).

2.4. Rational parametrization of (2.5). Use the coordinates $y_{1}$ and $y_{2}$ at the end of $\S 1.4$. [DF90a, Lemma 3.18] produces a rational point $\boldsymbol{m} \in \overline{\mathcal{C}}_{\alpha, \beta, s, t}$ over $z_{2}:\left(y_{1}+y_{2}\right)(\boldsymbol{m})=2 c$ and $\left(y_{1} y_{2}\right)(\boldsymbol{m})=c^{2}$. Equivalently,

$$
\begin{gathered}
U(\boldsymbol{m})=\left(y_{1}+y_{2}\right)(\boldsymbol{m})=U_{0}=2 c \text { and } \\
V(\boldsymbol{m})=\left(y_{1} y_{2}-5 c d\right) /\left(y_{1}+y_{2}\right)(\boldsymbol{m})=V_{0}=\frac{c^{2}-5 c d}{2 c}=\frac{c-5 d}{2} .
\end{gathered}
$$

Here is how $\boldsymbol{m}$ appears in the fiber product construction of $\S 1.4$.

Regard $\overline{\mathcal{C}}_{\alpha, \beta, s, t}$ as the normalization of one of the fiber products $Z_{p}=$ $\mathbb{P}_{y_{1}}^{1} \times_{\mathbb{P}_{z}^{1}} \mathbb{P}_{y_{2}}^{1}$ modulo the $S_{2}$ action. Let $y^{\prime}$ be the unique ramified point over $z_{2}$ in the degree 5 cover $f_{\boldsymbol{p}}: \mathbb{P}_{y}^{1} \rightarrow \mathbb{P}_{z}^{1}$. Then, $\boldsymbol{m}$ is the preimage of $\left(y^{\prime}, y^{\prime}\right)$.

Let $w$ be the parameter for tangent directions in $\mathbb{P}^{2}$ at the point $\boldsymbol{m}$. Euler's parametrization writes $U=w t+U_{0}, V=t+V_{0}$. Solve for the value of $t \neq 0$ giving an intersection different from $\left(U_{0}, V_{0}\right)$ with the conic if there is one. This displays coordinates for the conic as a function of $w$ :

$$
\begin{aligned}
& U(w)=\frac{8 c w^{2}+(-14 c+10 d) w+3 c-25 d}{4\left(w^{2}-3 w+1\right)}, \quad \text { and } \\
& V(w)=\frac{12 c w^{2}+(-11 c+5 d) w+2(c-5 d)}{4\left(w^{2}-3 w+1\right)} \quad \text { where } w=\frac{V-\frac{c-5 d}{2}}{U-2 c} .
\end{aligned}
$$


Start with $z$ as a function of $U$ and $V$ [DF90a, proof of Prop. 4.1] and rewrite $\bar{\Psi}_{\alpha, \beta, s, t}$ using $w$ :

$$
\begin{aligned}
z= & \frac{\alpha}{2}\left[\left(U^{5} / 5-U^{4} V+U^{3} V^{2}\right)-\frac{c+d}{4}\left(U^{4}-4 U^{3} V+2 U^{2} V^{2}\right)\right. \\
& \left.+c d\left(-3 U^{3}+4 U^{2} V\right)+\frac{5}{2} c d(c+d) U^{2}+\frac{25}{2}(c d)^{2}(c+d)\right]+\beta .
\end{aligned}
$$

2.5. Values of $z(w)$ (from $h_{\boldsymbol{p}}(w)$ in $\left(\mathrm{P}_{4}\right)$ ). Fix a fractional ideal $\mathcal{A}=\delta \mathbb{Z}$ of $\mathbb{Q}$. Make the following further assumption:

(2.8) $\alpha, \beta, c, d, \frac{\alpha}{10 \delta}$ and $\frac{\beta}{\delta}$ are integers.

The next two results complete the proof of Main Prop. 1.3 (and Main Theorem 1.2). Substitute (2.6) in (2.7) to complete computing $z(w)$.

Proposition 2.4. As $w$ runs over $\mathbb{Q}, z(w)$ takes on infinitely many values in $\mathcal{A}$. Thus, the subset of $\mathcal{H}_{\infty}(\mathbb{Q})$ satisfying the conclusion of Prop. 1.3 is Zariski dense.

Proof. Write $w$ as $m / n$ with $m, n \in \mathbb{Z}$. Then (2.6) becomes

$$
\begin{aligned}
& U=\frac{8 c m^{2}+(-14 c+10 d) m n+(3 c-25 d) n^{2}}{4\left(m^{2}-3 m n+n^{2}\right)}, \text { and } \\
& V=\frac{12 c m^{2}+(-11 c+5 d) m n+2(c-5 d) n^{2}}{4\left(m^{2}-3 m n+n^{2}\right)} .
\end{aligned}
$$

Rewrite $m^{2}-3 m n+n^{2}$ as $\left(m-\frac{3 n}{2}\right)^{2}-\frac{5 n^{2}}{4}$. The fundamental unit of $\mathbb{Q}(\sqrt{5})$ is $(1+\sqrt{5}) / 2$. Its 6 th power is $\theta=9+4 \sqrt{5}$. The components $(u, v)$ of powers of $\theta$ give integer solutions of $u^{2}-5 v^{2}=1$, with $v \equiv 0 \bmod 4$. Each such solution $(u, v)$ gives a solution $(m, n) \in \mathbb{Z}^{2}$ of $m^{2}-3 m n+n^{2}=1$ with $n \equiv 0 \bmod 4$. Further, with this holding, there are infinitely many $(m, n)$ for which (2.8) forces $U(\mathrm{~m} / n)$ to be a multiple of $2, V(\mathrm{~m} / n)$ to be an integer and $z(m / n) \in \delta \mathbb{Z}$.

Use the map $\varphi^{*}: \mathcal{P} \rightarrow \mathcal{H}_{\infty}$ from $\S 2.3$. Let $\mathcal{O}^{\prime}$ be the open subset of $\mathcal{P}$ consisting of 4 -tuples $(\alpha, \beta, c, d)$ mapping by $\varphi^{*}$ to $(\alpha, \beta, c+d, c d) \in \mathcal{H}_{\infty}$ [DF90a, §4.1]. Further, let $\Omega^{\prime}$ be the subset of $\mathcal{O}^{\prime}$ satisfying (2.8).

Then $\Omega^{\prime}$ is Zariski dense in $\mathcal{O}^{\prime}$. Further, points of $\Omega^{\prime}$ give a cover $\overline{\mathcal{C}}_{\alpha, \beta, s, t} \rightarrow$ $\mathbb{P}_{z}^{1}$ for which the conclusion of the Main Theorem holds. The image $\Omega^{\prime}$ in $\mathcal{H}_{\infty}$ is clearly Zariski dense. To finish only requires showing (1.14c) holds. The next proposition does exactly that.

Proposition 2.5. Suppose $f=f_{\boldsymbol{p}}$ corresponds to $\boldsymbol{p} \in \mathcal{P}(\mathbb{Q})$ as above. Then, $h_{f}(\mathbb{Q}) \cap f(\mathbb{Q}) \cap \mathcal{A}$ is finite.

Proof. We show $h_{f}(\mathbb{Q}) \cap f(\mathbb{Q}) \cap \mathcal{A}$ is finite. This means showing the normalization $W$ of the fiber product $\mathbb{P}_{y}^{1} \times_{\mathbb{P}_{z}^{1}} \mathbb{P}_{w}^{1}$ has only finitely many $\mathbb{Q}$ points over values of $z$ in $\mathcal{A}$. Siegel's own result shows this if each absolutely irreducible component of the fiber product has genus at least 1 . The remainder 
of the proof shows there are two such components, one of genus 1 , the other of genus 2.

Since $W$ covers $\mathbb{P}_{y}^{1}$, components of $W$ correspond to classes of conjugate points on $W$ over a generic point $y$ on $\mathbb{P}_{y}^{1}$. Points on $W$ over $y$ have the form, $\left(y, w_{i}\right), i=1, \ldots, 10$. Let $H$ be the stabilizer of 1 in the representation $R: S_{5} \rightarrow S_{10}$ of $S_{5}$. Galois theory corresponds components of $W$ to orbits of $H$ in the representation $R$. There are two orbits: $\left\{s_{1}^{\prime}, \ldots, s_{4}^{\prime}\right\}$; and the complement of this set of symbols from $\S 2.2$. Label the corresponding components as $W_{1}$ and $W_{2}$.

Abhyankar's Lemma in $\S 7$ lets us list points from $\mathbb{P}_{y}^{1}$ ramified in $W$ :

(2.9a) The unique value $y_{1}$ unramified over $z_{1}$;

(2.9b) the three points $y_{2, j}, j=1,2,3$, unramified over $z_{2}$; and

(2.9c) the analogous three points $y_{3, k}, k=1,2,3$, unramified over $z_{3}$.

Branch cycles for these points are conjugates, fixing 1 in the standard representation of $S_{5}$, of branch cycles for the corresponding points $z_{i}, i=1,2,3$. This information suffices to apply the Riemann-Hurwitz formula to $W_{i} / \mathbb{P}_{y}^{1}$.

So, $2\left(4+g\left(W_{1}\right)-1\right)=e_{1}+e_{2}+e_{3}: e_{1}$, the contribution from $y_{1}$, is $2 ; e_{2}$, from the three points over $z_{2}$, is 3 ; and so is $e_{3}$. Thus, $g\left(W_{1}\right)$ is 1 .

Here is the cycle type of the action of the branch cycles for $W_{2}$ on the symbols, $s_{j}^{\prime}, j=5, \ldots, 10$. (Use only their subscripts to identify them.)

(2.10a) (23)(45) acts on the last six symbols as (69)(78).

(2.10b) (23) acts as (68)(79).

Similar to above: $2\left(6+g\left(W_{2}\right)-1\right)=2+3 \cdot 2+3 \cdot 2=14$, or $g=2$.

\section{Completion of the Main Theorem with comments.}

$\S 3.1$ analyzes the totally arithmetic aspects of the proof of Thm. 1.2. $§ 3.2$ has exceptional degree 5 polynomials giving covers of $\mathbb{P}^{1}$ with only two finite branch points. Treating these in detail completes a missing item from the Main Theorem.

3.1. Comments on the proof of Thm. 1.2. Preliminaries to Thm. 1.2 used branch cycle description and the group theory of Nielsen classes. Reducing computations to group theory can be more efficient than manipulating equations. Hurwitz type families support translation to group theory because they have a natural moduli definition. Thus, they include all appropriate covers even when it would be prohibitive to write equations for these. [DF90a] (and related papers) suggest how to detect diophantine properties of members of these families from group theory alone. You never see the coefficients of the equations, so their complexity is irrelevant. Thm. 1.2 includes a diophantine question we haven't yet reduced to group theory. The following remarks discuss related points in the proof. 
Remark 3.1. The highest homogeneous part of conic (2.4) (in $U$ and $V$ ) is constant in $c$ and $d$. [DF90a, p. 135] finds $U$ and $V$ as generators of the linear system of the divisor $\boldsymbol{m}_{1}+\boldsymbol{m}_{2}$ on $\mathcal{T}_{5, \boldsymbol{p}}^{(2)}$ over $z=\infty$. The fiber product construction and Abhyankar's Lemma ( $(7)$ trace this to a property of the Puiseux expansions for the cover $f_{\boldsymbol{p}}: \mathbb{P}_{y}^{1} \rightarrow \mathbb{P}_{z}^{1}$ around $z=\infty$. It is that a constant field extension, $\mathbb{Q}\left(\zeta_{5}\right)$, over the coordinates for $\boldsymbol{p}$ contains the coefficients of these expansions.

Remark 3.2. Eulerian substitution in (2.4) replaces $U$ by $w t+U_{0}$ and $V$ by $t+V_{0}$. Then solve (2.4) for $t \neq 0$ giving the other intersection of this line through $\left(U_{0}, V_{0}\right)$ with the conic. Since $\left(U_{0}, V_{0}\right)$ are polynomials in $c$ and $d$, Remark 3.1 implies $c$ and $d$ appear only in the numerators of $U(w)$ and $V(w)$ from (2.6). The functions $U=y_{1}+y_{2}$ and $V=\left(y_{1} y_{2}-5 c d\right) /\left(y_{1}+y_{2}\right)$ generate the function field of $\mathcal{T}_{5, \boldsymbol{p}}^{(2)}$. Their polar divisors have support in the divisor on $\mathcal{T}_{5, \boldsymbol{p}}^{(2)}$ over $z=\infty$. Thus, $z$ in $(2.7)$ is a polynomial in all nonconstant variables excluding $w$. So, the denominator of $z$ is a power of the denominator of $U$ (and $V$ ). These conclusions therefore come from Remark 3.1 and that $\left(U_{0}, V_{0}\right)(\S 2.4)$ are polynomials in $c$ and $d$. Will this hold generally when ramification provides rational points (the major theme of $[$ DF90a $])$ ?

Remark 3.3. Expression $4\left(w^{2}-3 w+1\right)$ appears in the denominator for $z$. Congruence conditions (2.8) cancel the appearance of 4 . Further, when we set $w=m / n$ and homogenize, there are integers $m, n$ making the denominator of $z$ equal to 1 . In similar situations expect these to hold by adding congruence conditions like those of (2.8). This assumes Remark 3.1 and 3.2 conditions generalize.

3.2. Main Theorem 1.2 three branch point specializations. This section produces branch cycles for polynomial covers $v: \mathbb{P}_{y}^{1} \rightarrow \mathbb{P}_{z}^{1}$ that satisfy (1.13b) in Main Theorem 1.2. The argument for Theorem 1.1 applies, except these covers have only two finite branch points, instead of three. As in $\S 1.5, R: S_{5} \rightarrow S_{10}$ is the degree 10 representation of $S_{5}$. As before, $z=\infty$ totally ramifies and branch cycles $\left(g_{1}, g_{2}, g_{3}\right)=\boldsymbol{g}$ satisfy the following.

(3.1a) $g_{3}$ is a 5 -cycle corresponding to total ramification over $\infty$.

(3.1b) $g_{1} g_{2} g_{3}=1$ and $\left\langle g_{1}, g_{2}, g_{3}\right\rangle=A_{5}$ or $S_{5}$.

(3.1c) With $\boldsymbol{g}^{\prime}=R(\boldsymbol{g})$, Riemann-Hurwitz shows $\boldsymbol{g}$ and $\boldsymbol{g}^{\prime}$ give genus zero covers.

The 3-tuples $\boldsymbol{g}^{\prime}$ are branch cycles for the cover we previously were calling $h_{v}$. As in (1.11), (3.1c) means ind $\left(g_{1}\right)+\operatorname{ind}\left(g_{2}\right)=4$ and $\operatorname{ind}\left(g_{1}^{\prime}\right)+\operatorname{ind}\left(g_{2}^{\prime}\right)=10$. For example, if ind $\left(g_{i}\right)=2$, then $g_{i}$ is either a 3-cycle or a product of two disjoint 2 -cycles, $i=1,2$. With $g_{3}=(54321)$, possible branch cycles for 
$\left(g_{1}, g_{2}\right)$ are

$$
((123),(145)),((135),(23)(45)) \text {. }
$$

Reject another apparent possibility: ((12)(35),(13)(45)). As both $g_{1}$ and $g_{2}$ are involutions, they generate the dihedral group of degree 5 , not $A_{5}$ or $S_{5}$.

The (3.2) examples gives $\left(g_{1}, g_{2}\right)$ generating $A_{5}$. The next requirement is

$$
\operatorname{ind}\left(g_{1}^{\prime}\right)+\operatorname{ind}\left(g_{2}^{\prime}\right)=10 \text {. }
$$

This interprets that $h_{v}$ (notation as in $\S 1.6$ for $h_{f}$ ) is of genus 0 . As in $\S 1.5$, for $g$ a 2-cycle, $R(g)$ has shape $(2)(2)(2)$; for $g$ a product of disjoint 2-cycles, $R(g)$ has shape $(2)(2)(2)(2)$; and for $g$ a 3-cycle, $R(g)$ has shape $(3)(3)(3)$. Only the second from (3.2) satisfies (3.3). That is, suppose $v$ is a polynomial cover with branch cycles from the first entry in (3.2). Then, instead of giving $h_{v}$ as in Main Prop. 1.3, it produces a genus 1 cover. (Note: This is a coalescing from List B of $\S 4.4$.)

The other possibility from $\operatorname{ind}\left(g_{1}\right)+\operatorname{ind}\left(g_{2}\right)=4$ is that one of $\operatorname{ind}\left(g_{i}\right)$ is 1 , the other $3, i=1,2$. We might have $g_{1}$ a 2-cycle and $g_{2}$ a 4-cycle; or $g_{1}$ a 2-cycle and $g_{2}$ a product of disjoint 2 and 3-cycles. Here are examples:

$$
((12),(1345)),((13),(23)(145)) \text {. }
$$

Both branch cycle descriptions generate $S_{5}$. If $g$ is a 4-cycle, then $R(g)$ has shape $(2)(4)(4)$, and if $g$ has shape $(2)(3)$, then $R(g)$ has shape $(6)(3)$. Both pass the genus 0 condition for $R(\boldsymbol{g})$. The next result amends Main Th. 1.2 by showing these branch cycles have a corresponding $\left(v, h_{v}\right)$ over $\mathbb{Q}$.

Lemma 3.4 (Completion Lemma). The polynomials in (1.13b) of Main Theorem 1.2 consist of $v_{1}, v_{2}, v_{3} \in \mathbb{Q}[y]$ (with explicit branch cycle descriptions in the proof below). That is, suppose $f \in S_{\mathcal{A}}$ for some fractional $\mathbb{Q}$ ideal, (1.13a) does not hold, and $f: \mathbb{P}_{y}^{1} \rightarrow \mathbb{P}_{z}^{1}$. Then, there are affine functions $\alpha, \beta$ for which $f(y)=\beta\left(v_{i}(\alpha(y))\right)$ for some value of $i$ from $\{1,2,3\}$.

Proof. From the proof of the Main Proposition, it suffices to find three polynomials $v_{1}, v_{2}, v_{3}$ giving each $f$ satisfying (3.1) by the affine change (from the statement of the lemma). Suppose the finite branch points of $f$ are $z_{1}$ and $z_{2}$. The above discussion shows, beyond (3.1), the major properties of any branch cycle description for $f$. In each case the finite branch cycles are in distinct conjugacy classes of $N_{S_{5}}\left(A_{5}\right)=S_{5}$. So, $z_{1}, z_{2} \in \mathbb{Q}$. (For this and non-Galois cover application of rigidity, see the branch cycle argument in [Fri95a, §3] or the source of that in [Fri77, start of §5].) If its finite branch cycles are $\left(g_{1}, g_{2}\right)-g_{i}$ corresponding to $z_{i}$ - then, one of these hold. Either: $g_{1}$ has shape (3) and $g_{2}$ shape $(2)(2) ; g_{1}$ has shape $(2)$ and $g_{2}$ shape (4); $g_{1}$ has shape (2) and $g_{2}$ shape (2)(3); or switching $g_{1}$ and $g_{2}$ gives these shapes. 
We now produce polynomials $v_{1}, v_{2}, v_{3}$ in $y$. These are unique up to affine change of $y$ and composition with one of the functions $z \mapsto z$ or $z \mapsto 1-z$ given the following conditions. They have branch cycle shapes at the points 0 and 1 as one of the first three above. As previously, take the branch cycle for $z=\infty$ to be $g_{3}=(12345)^{-1}$. Riemann's existence theorem produces corresponding covers, up to absolute equivalence, if the branch cycles equal one of

$$
\text { a) }((135),(23)(45)), b)((12),(1345)) \text { or } c)((13),(23)(145)) .
$$

This is a simple combinatorial computation.

Polynomials give the three covers because they are of genus 0 , the covering space has a rational point (the ramified point over 0 ) and $z=\infty$ totally ramifies $\left(g_{3}\right.$ is a 5 -cycle). Polynomials with coefficients in $\mathbb{Q}$ represent these covers because their equivalence class of branch cycles uniquely determines them. This is from the non-Galois cover application of rigidity [Fri77, Theorem 5.1].

Cases (3.4a) and (3.4b). For (3.4a), $g_{2}$ is a product of two disjoint 2cycles. Conjugate by a power of $g_{3}$, to assume up to absolute equivalence, the support of $g_{2}$ excludes 1 . We claim, $g_{1} g_{2}=(12345)$ determines $g_{1}$ and $g_{2}$. Write $g_{1}=(1 k j)$ and $g_{2}=\left(k k^{\prime}\right)\left(j j^{\prime}\right)$. Then, the claim follows from $g_{1} g_{2}=\left(1 k^{\prime} k j^{\prime} j\right)$. For Case $(3.4 b), g_{2}$ is a 4 -cycle. This is similar to $(3.4 a)$.

Case (3.4c). Here $g_{2}$ has shape (2)(3). The support of $g_{1}$ has common support in both the 2-cycle and the 3 -cycle of $g_{2}$. The branch cycles $\left(g_{1}, g_{2}\right)$ therefore look like this: $((1 i),(1 j)(i k l))$, and $g_{1} g_{2}=(1 k l i j)$. As in $(3.4 a)$, $g_{1} g_{2}=(12345)$ determines $g_{1}$ and $g_{2}$.

\subsection{Arithmetic of the polynomials in Completion Lemma 3.4. It} is easy to find a rational point on the degree 10 cover $\mathcal{T}_{(i)} \rightarrow \mathbb{P}^{1}$ arising from $v_{i}$ (of Lemma 3.4) as in $\S 1.5, i=1,2,3$. For example, the divisor of the fiber of $\mathcal{T}_{(1)} \rightarrow \mathbb{P}_{z}^{1}$ over 0 has shape (3)(3)(3). Therefore, $\mathbb{Q}$ is a field of definition of the unramified point in this fiber. For $j=2,3$, the divisor of the fiber of $\mathcal{T}_{(j)} \rightarrow \mathbb{P}_{z}^{1}$ over 0 has shape $(2)(2)(2)$. Thus, the set of 3 ramified points is a $\mathbb{Q}$ divisor. As in $\S 2.1$, this produces a $\mathbb{Q}$ point from ramification. Conclude the following.

Proposition 3.5. There is a $\mathbb{Q}$ rational function $h_{v_{i}}: \mathbb{P}_{w}^{1} \rightarrow \mathbb{P}_{z}^{1}$ giving a cover equivalent to $\mathcal{T}_{(i)} \rightarrow \mathbb{P}_{z}^{1}, i=1,2,3$.

Now we explain the meaning that these $v_{i}$ s are coalescings from the polynomials of Thm. 1.1. Algebraically it is that $v_{i}$ is a specialization of functions at the generic point of $\mathcal{H}_{\infty}$. This, however, is equivalent to saying a coalescing of branch cycles from $\mathrm{Ni}_{\infty}$ gives branch cycles for these covers. Combinatorially: Each $v_{i}$ has a branch cycle description from one of $\left(g_{1} g_{2}, g_{3}, g_{4}\right)$, 
$\left(g_{1}, g_{2} g_{3}, g_{4}\right)$ or $\left(g_{1}, g_{2}, g_{3} g_{4}\right)$ where $\left(g_{1}, g_{2}, g_{3}, g_{4}\right) \in \mathrm{Ni}_{\infty}$. $(\S 4.3$ discusses a general definition for coalescing of branch cycles.) Here are examples.

Coalesce the first and second branch cycles in $((23)(45),(12),(14))$. This gives $((123)(45),(14))$. These are the finite branch cycles of a polynomial cover. Let $\alpha(z)=1-z$. This cover is equivalent to $\alpha \circ v_{3}: \mathbb{P}_{y}^{1} \rightarrow \mathbb{P}_{z}^{1}$. Similarly, coalesce the second and third branch cycles in $((23)(45),(12),(14))$ to get $((23)(45),(124))$ for finite branch cycles of a cover equivalent to $\alpha \circ v_{1}: \mathbb{P}_{y}^{1} \rightarrow \mathbb{P}_{z}^{1}$.

Finally, coalesce the first and second branch cycles in $((25)(34),(35)$, (12)) to get $((2345),(12))$, finite branch cycles for a cover equivalent to $\alpha \circ v_{2}: \mathbb{P}_{y}^{1} \rightarrow \mathbb{P}_{z}^{1}$. Theoretically it requires complicated base change of coordinates to see this specialization. An argument for this is in [Fri95c, §III.F]: The discussion of specialization sequences. Here, however, coordinates for the space $\mathcal{P}$ of $\S 2.3$ suffice and it is easy to see the coalescing corresponds to specialization of variables for $\mathcal{P}$.

There is a way to view coalescing as coming from paths. Suppose a description of branch cycles $\left(g_{1}, g_{2}, g_{3}, g_{4}\right)$ comes from a choice of paths $P_{1}, P_{2}, P_{3}, P_{4}$ on the sphere punctured at four points. Then, there is a natural set of paths to attach to coalescing these branch cycles to $\left(g_{1} g_{2}, g_{3}, g_{4}\right)$ : $P_{1} \cdot P_{2}, P_{3}, P_{4}$. That is, you replace the first two paths by their product. Do this and then choose a homotopy of the product $P_{1} \cdot P_{2}$ to a loop $P^{\prime}$ enclosing the branch points $z_{1}$ and $z_{2}$. Keep $P^{\prime}$ fixed and let the branch points come together complex analytically. This choice, for example, preserves the complex conjugation operator of Real Point Lemma 2.1.

We haven't ruled out that one from the $v_{i}$ s may be in $S_{\mathcal{A}}$ for each fractional ideal $\mathcal{A}$. Suppose for example, $v=f(x) / u(x)^{t}$ with $f, u \in \mathbb{Z}[x]$ monic polynomials, $\operatorname{deg}(u)=2$ has real roots $\alpha_{1}, \alpha_{2}$ conjugate over $\mathbb{Q}$ and $\operatorname{deg}(f)=2 t$. Assume $\epsilon=r_{1}-\alpha_{1} r_{2}$ is the fundamental unit of $\mathbb{Q}\left(\alpha_{1}\right)$ with $s_{1}, s_{2} \in \mathbb{Q}$, and $\epsilon^{s}=m_{s}-\alpha_{1} n_{s}$. An arbitrary such $v$ might satisfy the following: $v(x) \equiv 0 \bmod p^{e}$ has a solution for each prime $p$ and each positive integer $e$. This happens if $f(x)$ has a linear factor over $\mathbb{Q}$. There are other cases too.

For example, consider $f(x)=h(x)\left(x^{2}-2\right)\left(x^{2}-3\right)\left(x^{2}-6\right)$ with $h(x)$ having a 2 -adic and a 3 -adic zero, neither in $\mathbb{Q}$. For such an $f$, the Chinese Remainder Theorem produces for $f(x) \equiv 0 \bmod w$ a solution for each integer $w$. Here, however, assuring the denominator equals \pm 1 , requires for each prime power $p^{e}$ there is an $s$ with $n_{s}^{2 t} f\left(m_{s} / n_{s}\right) \equiv 0 \bmod p^{e}$ having a solution.

Problem 3.6. Suppose $h: \mathbb{P}_{w}^{1} \rightarrow \mathbb{P}_{z}^{1}$ is a $\mathbb{Q}$ rational function with exactly two real conjugate (geometric) points over $z=\infty$. Is there a nontrivial fractional ideal $\mathcal{A}$ with $h \notin S_{\mathcal{A}}(\S 1.4)$ ? 


\section{Siegel and Néron families.}

$\S 4.1$ defines Siegel families and $\S 4.2$ lists many examples. $\S 4.4$ comments briefly by example on what we call Néron families. This is an analog of Siegel families of covers, except the covers $\mathcal{T}_{\boldsymbol{p}} \rightarrow \mathbb{P}_{z}^{1}$ (like those of $\S 1.4$ ) for $\boldsymbol{p} \in \mathcal{P}$ are by curves of genus 1 . The arithmetic assumption is that $\mathcal{P}(\mathbb{Q})$ is Zariski dense in $\mathcal{P}$. The arithmetic question is whether $\mathcal{T}_{\boldsymbol{p}}(\mathbb{Q})$ is infinite for a dense set of $\boldsymbol{p} \in \mathcal{P}(\mathbb{Q})$. The principal example of $\S 4$.4 resembles that of Main Theorem 1.2: Does some Néron family of covers contribute to $\mathcal{R}_{f}(\mathbb{Q}) \backslash \mathcal{V}_{f}(\mathbb{Q})$ being infinite.

4.1. Definition of Siegel families. Let $\mathrm{Ni}(G, \mathbf{C}, R), \mathbf{C}=\left(\mathrm{C}_{1}, \ldots, \mathrm{C}_{r}\right)$, be a Nielsen class (as in $\S 1.1$ ) with $R: G \rightarrow S_{m}$ a degree $m$ faithful representation of $G$. Assume for $g \in \mathrm{C}_{r}, R(g)$ is a product of two disjoint $m / 2$-cycles. Consider a smooth irreducible family $\Psi: \mathcal{T} \rightarrow \mathcal{P} \times \mathbb{P}_{z}^{1}$ of curve covers over $\mathbb{Q}$ with degree $m$ fibers $\Psi_{\boldsymbol{p}}: \mathcal{T}_{\boldsymbol{p}} \rightarrow \mathbb{P}_{z}^{1}$ in $\mathrm{Ni}(G, \mathbf{C})$ with geometric monodromy group $G$ and associated representation $R$. If the following properties hold, we say $\Psi$ is a Siegel family (in the Nielsen class $\operatorname{Ni}(G, \mathbf{C})$ ).

(4.1a) For each $\boldsymbol{p} \in \mathcal{P}, z=\infty$ in $\Psi_{\boldsymbol{p}}: \mathcal{T}_{\boldsymbol{p}} \rightarrow \mathbb{P}_{z}^{1}$ has associated branch cycle in $\mathrm{C}_{r}$.

(4.1b) For each $\boldsymbol{p} \in \mathcal{P}, \mathcal{T}_{\boldsymbol{p}}$ has genus 0 .

(4.1c) For a Zariski dense subset of $\boldsymbol{p} \in \mathcal{P}(\mathbb{Q})$, the (geometric) support points $y_{1, \boldsymbol{p}}, y_{2, \boldsymbol{p}}$ of $\Psi_{\boldsymbol{p}}^{-1}(\infty)$ are real and conjugate over $\mathbb{Q}$.

The Hurwitz space $\mathcal{H}(G, \mathbf{C}, R)^{\text {abs }}$ is always available. Denote the quotient of $\left(\mathbb{P}^{1}\right)^{r} \backslash \Delta_{r}$ by the stabilizer of $r$ in $S_{r}(\S 6.2)$ by $U_{S_{r-1}}$. Label the subset of $U_{S_{r-1}}$ with $\infty$ in the $r$ th coordinate as $U_{S_{r-1}, \infty}$. Then, points of the fiber product

$$
\mathcal{H}_{\infty}^{\mathrm{abs}}=\mathcal{H}(G, \mathbf{C}, R)^{\mathrm{abs}} \times_{\mathbb{P}^{r} \backslash D_{r}} U_{S_{r-1}, \infty}
$$

represent covers of $\mathbb{P}_{z}^{1}$ in the Nielsen class $\mathrm{Ni}(G, \mathbf{C})$. These automatically inherit properties (4.1a) and (4.1b). The shape of $\mathrm{C}_{r}$ guarantees covers of $\mathbb{P}_{z}^{1}$ representing $\boldsymbol{p}^{\prime} \in \mathcal{H}_{\infty}^{\text {abs }}$ have two points $y_{1, \boldsymbol{p}^{\prime}}, y_{2, \boldsymbol{p}^{\prime}}$ of ramification index $m / 2$ lying over $\infty$. Call a Siegel family complete if the natural map $\mathcal{P} \rightarrow \mathcal{H}(G, \mathbf{C})_{\infty}^{\text {abs }}$ is birationally surjective on an absolutely irreducible component of $\mathcal{H}(G, \mathbf{C})_{\infty}^{\text {abs }}$. Then, condition (4.1c) produces a dense set of $\boldsymbol{p}^{\prime} \in \mathcal{H}(G, \mathbf{C})_{\infty}^{\text {abs }}(\mathbb{Q})$ for which $y_{1, \boldsymbol{p}^{\prime}}$ and $y_{2, \boldsymbol{p}^{\prime}}$ are real and conjugate over $\mathbb{Q}$. Let $G(1)$ be the subgroup of elements $g \in G$ for which $R(g)$ fixes the integer 1 of the representation. Recall: $\S 1.3$ notes $\mathcal{H}(G, \mathbf{C})_{\infty}^{\text {abs }}$ supports a unique total family $\mathcal{T}$ of representing covers if and only if $G(1)$ is self-normalizing in $G$. Still, even without this assumption, working with complete families returns us to properties of $\mathcal{H}(G, \mathbf{C})_{\infty}^{\text {abs }}$.

4.2. Siegel families with $r$ large. There are probably too many Siegel families to classify them. Further, it is difficult - though as we are showing, sometimes possible - in practice to know if $\mathcal{P}$ has a Zariski dense set 
of $\mathbb{Q}$ points (property (4.1c)). This subsection gives several examples of Siegel families. For any Siegel family, one can ask if the conclusion of Main Theorem 1.2 holds: Is

$$
\left\{\boldsymbol{p} \in \mathcal{P}(\mathbb{Q})|| \Psi_{\boldsymbol{p}}\left(\mathcal{T}_{\boldsymbol{p}}(\mathbb{Q})\right) \cap \mathcal{A} \mid=\infty\right\}
$$

Zariski dense? Also, is this a family of rational functions $(\S 1.7)$ ?

The easiest complete Siegel family has $G=S_{m}, R$ the standard representation and $m=2 k$. Take $\mathcal{H}_{\infty}(G, \mathbf{C})_{\infty}^{\text {abs }}$ where $r=m+1, \mathbf{C}=\left(\mathrm{C}_{1}, \ldots, \mathrm{C}_{m+1}\right)$ has $\mathrm{C}_{m+1}$ with cycle type $(k)(k)$ and $\mathrm{C}_{i}, i=1, \ldots, m$, is the conjugacy class of 2-cycles. Pullback of $\mathcal{H}_{\infty}(G, \mathbf{C})_{\infty}^{\text {abs }}$ to an appropriate analog of $\mathcal{P}$ as in $\S 2.3$ produces a family of rational functions of form $f(w)=M(w) /\left(w^{2}+c w+d\right)^{k}$. Here, $M(w)$ is a polynomial of degree at most $2 k$, and the zeros of $M(w)$ and of $w^{2}+c w+d$ are all distinct. It simplifies to assume - a choice we can make canonically - that $\operatorname{deg}(M(w)) \leq 2 k-1$. An additional condition assures $f$ gives a cover in the Nielsen class:

(4.2) $\frac{d M}{d w}\left(w^{2}+c w+d\right)-M(w)(2 w+c)=0$ has distinct zeros and these have $2 k$ distinct images under $f$.

This includes the case $M(w)$ is a general polynomial of degree $m-1$ and $c, d$ are also general. The field of definition of the points $w_{1}$ and $w_{2}$ over $\infty$ varies with $\boldsymbol{p} \in \mathcal{P}$. This is because zeros of $w^{2}+c w+d$ vary with $c$ and $d$ and these give coordinates for the $w_{i}$ s. It is easy to see condition (4.1c) holds. There is a Zariski dense set of $c$ and $d$ in $\mathbb{Q}$ with $w^{2}+c w+d$ having real conjugate zeros.

Here is a more serious example. The parameter space is $\mathcal{H}_{\infty}^{\text {abs }}=$ $\mathcal{H}\left(G, \mathbf{C}^{\prime}\right)_{\infty}^{\text {abs }}$ where $G=A_{n}$ for some integer $n=2 k \geq 6$ and $r=k+1$. Specific conjugacy classes are $\mathrm{C}_{r}^{\prime}$ with cycle type $(k)(k)$ and $\mathrm{C}_{i}^{\prime}, i=1, \ldots, r-1$, the class of 3 -cycles. $\S 4.3$ gives representative elements of $\mathrm{Ni}\left(A_{2 k}, \mathbf{C}^{\prime}\right)$. Some experience with this example comes from [Fri96]. This lists components of $\mathcal{H}\left(A_{n}, \mathbf{C}_{3^{r}}\right)$ for all $r$ and all $n \geq 5: \mathbf{C}_{3^{r}}$ is $r$ repetitions of the conjugacy class of 3 -cycles. When $r=n-1$, there is one absolutely irreducible component (defined over $\mathbb{Q}$ ).

[Mes90] uses rational functions with square discriminant. For $n$ odd, this produces a unirational family of rational function covers over $\mathbb{Q}$ having $r=n-13$-cycles as branch cycles. As [Fri96] gives only one component in this case, $\mathcal{H}\left(A_{n}, \mathbf{C}_{3^{n-1}}\right)$ is unirational. Here, though, we are in the case $n$ is even. In the next remark, we produce elements of $\mathrm{Ni}\left(A_{2 k}, \mathbf{C}^{\prime}\right)$ satisfying the conditions above. Yet, the Hurwitz spaces may not give Siegel families, because we don't know if they have a dense set of $\mathbb{Q}$ points.

\subsection{Producing elements of $\mathrm{Ni}\left(A_{2 k}, \mathbf{C}^{\prime}\right)$ by coalescing Nielsen clas-} ses. Let $\operatorname{Ni}(G, \mathbf{C})$ and $\operatorname{Ni}\left(G^{*}, \mathbf{C}^{*}\right)$ be Nielsen classes with $\mathbf{C}$ an $r$-tuple and $\mathbf{C}^{*}$ an $r^{*}$-tuple of conjugacy classes from respective groups $G$ and $G^{*}$. Assume $P$ partitions $\left\{1, \ldots, r^{*}\right\}$ into $r$ sets, each consisting of contiguous integers. Example: If $r^{*}=5$ and $r=4, P=\{1\} \cup\{2\} \cup\{3\} \cup\{4,5\}$ is such 
a partition. Given $P$, there is a natural map

$$
\mathrm{Ni}\left(G^{*}, \mathbf{C}^{*}\right) \rightarrow\left\{\boldsymbol{g} \in\left(G^{*}\right)^{r} \mid g_{1} \cdots g_{r}=1\right\}
$$

by $\boldsymbol{g}^{*} \mapsto\left(g_{1}, \ldots, g_{r}\right) ; g_{i}$ is the product in order of entries whose subscripts are in the $i$ th term of the partition $P$. Use $P$ also to denote this map. We say, $\boldsymbol{g} \in \operatorname{Ni}(G, \mathbf{C})$ is in the $P$ boundary of $\operatorname{Ni}\left(G^{*}, \mathbf{C}^{*}\right)$ if there exists $\boldsymbol{g}^{*} \in \operatorname{Ni}\left(G^{*}, \mathbf{C}^{*}\right)$ with $P\left(\boldsymbol{g}^{*}\right)=\boldsymbol{g}$. Write $\operatorname{Ni}(G, \mathbf{C})$ as a union $O_{1} \cup O_{2} \cup \cdots \cup O_{t}$ of braid orbits $(\S 1.2)$. If $\boldsymbol{g} \in O_{u}$, then say $O_{u}$ is in the $P$ boundary of $\operatorname{Ni}\left(G^{*}, \mathbf{C}^{*}\right)$.

4.3.1. Case: $k=3, r=5$. From [Fri96],

$$
\boldsymbol{g}=\left((123),(134),(142),(156),(156)^{-1}\right)
$$

is in the unique braid orbit on $\operatorname{Ni}\left(A_{6}, \mathbf{C}_{3^{5}}\right)$. The first three entries of $\boldsymbol{g}$ are transitive on $\{1,2,3,4\}$; the last two have product 1 . So, the Blocks Lemma [Fri96, §1.3] produces a braid conjugating the last two entries by $(14)(23)$ (fixed on the other entries). Then, braid to ((465), (123), (134), (142), (456)). Finally, braid (123) unchanged past the two 3-cycles to its right. This gives

$$
\text { ((465), (142), (243), (123)(456)): }
$$

A representative of $\mathcal{H}\left(A_{6}, \mathbf{C}^{\prime}\right)$ for this case. Here $C=(23)(45)$ in the Real Point Lemma 2.1. This gives a cover where the two points over $\infty$ are real.

4.3.2. Case: $k=4 r=7$. The unique braid orbit of $\mathrm{Ni}\left(A_{8}, \mathbf{C}_{3^{7}}\right)$ contains

$$
\text { ((123), (134), (142), (156), (651), (178), (187)). }
$$

If there is an analog of the case $k=3$, there is a product of three 3-cycles giving cycle type (4)(4) over the special branch point. Yet, no element of $\operatorname{Ni}\left(A_{8}, \mathbf{C}_{3^{7}}\right)$ coalesces to an element of $\mathcal{H}\left(A_{8}, \mathbf{C}^{\prime}\right)$. Argument: Suppose a product of three 3 -cycles has shape $(4)(4)$. Then, all eight integers appear among the nine places of support in the three 3-cycles. Only one integer (say, 1) can appear twice in the supports of the 3 -cycles. Up to reordering integers, the 3 -cycles must be $((123),(145),(678))$. Yet, this product has shape $(5)(3)$.

Here, however, is an element of $\mathrm{Ni}\left(A_{8}, \mathbf{C}^{\prime}\right)$ :

$$
\text { ((123), (145),(678),(165),(4321)(8765)). }
$$

Apply Real Point Lemma 2.1 to see if it gives a cover with real points over $\infty$. (Some element of this Nielsen class must have this property for this to be a Siegel family.) The complex conjugation operator takes (4.3) to

$$
\text { ((132), (354),(687), (485),(1234)(5678)). }
$$

So, if $C$ exists, it conjugates (4.3) to (4.4). The 1st, 3rd and 5th entries force $C$ to be $(13)(68)$. This doesn't, however, conjugate the 2 nd and 
fourth entries of (4.3) to the corresponding entries of (4.4). That leaves a programming question.

Problem 4.1. Does $\operatorname{Ni}\left(A_{8}, \mathbf{C}^{\prime}\right)$ contain branch cycles for a cover (corresponding to Lemma 2.1) with two real points over $\infty$ ?

4.3.3. Case: general $n=2 k, k \geq 5$ odd. Here is a representative of $\mathrm{Ni}\left(A_{2 k}, \mathbf{C}_{3^{2 k-1}}\right)$ :

$$
\begin{gathered}
\left((123),(134),(142),(156),(156)^{-1},(178),(178)^{-1},\right. \\
\left.(12 k-12 k),(12 k-12 k)^{-1}\right)
\end{gathered}
$$

As with $n=6$, a braid conjugates

$$
\left((1 k+2 k+3),(1 k+2 k+3)^{-1}, \ldots,(12 k-12 k),(12 k-12 k)^{-1}\right)
$$

by $(14)(23)$ without changing anything else. This changes these $(k-$ $1) / 2$ pairs to $\left((4 k+2 k+3),(4 k+2 k+3)^{-1}, \ldots,(42 k-1,2 k),(42 k-12 k)^{-1}\right)$. Braid the 1st, 3rd, 5th, etc. of these together to form $((4 k+2 k+3), \ldots$, $(42 k-12 k), *)$. Coalesce entries up to $*$. This gives a $k$-cycle on $\{4, k+2$, $k+3, \ldots, 2 k-1,2 k\}$. From the remaining 3-cycles, (123), (156),.., $(1 k k+1)$ appear from left to right in (4.5). Braid these together to the exact left of the previous $k$-cycle. Then, coalesce them to a $k$-cycle on $\{1,2,3,5, \ldots, k+1\}$. The two $k$-cycles have disjoint supports. Coalescing leaves an element of shape $(k)(k)$ and 3 -cycles. This shows how to coalesce an element of $\mathrm{Ni}\left(A_{2 k}, \mathbf{C}_{3^{k+1}}\right)$ to an element of $\mathrm{Ni}\left(A_{2 k}, \mathbf{C}^{\prime}\right)$.

4.4. Néron version of the problem. $\S 5.1$ has an update on results extending Main Theorem 1.2. This includes variants of the original problem. Example: Drop the restriction that $z_{0}$, with $f(y)-z_{0}$ reducible, must lie in a given fractional ideal. That is, replace the condition $f \in S_{\mathcal{A}}$ in $\S 1.4$ with $f \in S_{\mathbb{Q}}:$

(4.6) $f \in \mathbb{Q}[y]$ with $\mathcal{R}_{f}(\mathbb{Q}) \backslash \mathcal{V}_{f}(\mathbb{Q})$ infinite.

As in Thm. 1.2, $f \in S_{\mathbb{Q}}$ comes from a simple geometry setup. Let $\hat{\varphi}: \hat{X} \rightarrow$ $\mathbb{P}_{z}^{1}$ be the minimal Galois closure of $f: \mathbb{P}_{y}^{1} \rightarrow \mathbb{P}_{z}^{1}$. The cover $f$ produces a standard faithful permutation representation $T_{f}: G\left(\hat{X} / \mathbb{P}_{z}^{1}\right)=\hat{G} \rightarrow S_{n}$ with $n=\operatorname{deg}(f)$. Call a subgroup of $\hat{G}$ intransitive if its image in $S_{n}$ is an intransitive subgroup.

For each subgroup $H$ of $\hat{G}$ consider the quotient cover $\varphi_{H}: \hat{X} / H \rightarrow \mathbb{P}_{z}^{1}$. Let $\mathbb{H}$ run over maximal proper subgroups $H$ satisfying the following.

(4.7a) $\hat{X} / H$ has $\infty$-ly many $\mathbb{Q}$ points.

(4.7b) $H$ is intransitive.

One element of $\mathbb{H}$ is $G_{1}$, the subgroup defining the cover $f$. Remove from $\mathbb{H}$ groups containing conjugates of $G_{1}$. Denote the result by $\mathbb{H}^{\prime}$. The next result is in [Fri86] and [Fri77, Intro.]. 
Proposition 4.2. Suppose $f \in S_{\mathbb{Q}}$ and $f$ is indecomposable. Then $\mathcal{R}_{f}(\mathbb{Q}) \backslash$ $\mathcal{V}_{f}(\mathbb{Q})$ is the union of a finite set and

$$
\bigcup_{H \in \mathbb{H}^{\prime}} \varphi_{H}((\hat{X} / H)(\mathbb{Q})) .
$$

Remark 4.3 (Comments on Prop. 4.2). Suppose $f \in K[y]$ has degree prime to the characteristic of $K$. Then $f$ is indecomposable over $K$ if and only if it is indecomposable over $\bar{K}$ (see top of $\S 1$ ). From Faltings' Theorem, $f \in S_{\mathbb{Q}}$ requires there exists $H \in \mathbb{H}^{\prime}$ with $\hat{X} / H$ of genus 0 or 1 . [Ser92, §5.4] discusses a similar situation using Faltings' Theorem. Branch cycle descriptions of covers allow checking the genus of $\hat{X} / H$ by applying Riemann-Hurwitz. That is, let $G$ be the geometric monodromy group of the cover, $\boldsymbol{g}$ a branch cycle description of $f: \mathbb{P}_{y}^{1} \rightarrow \mathbb{P}_{z}^{1}$ and $T_{H}: G \rightarrow S_{(G: H)}$ the permutation representation on cosets of $H$. The genus of $\hat{X} / H$ is $g_{H}$ in

$$
2\left((G: H)+g_{H}-1\right)=\sum_{i=1}^{r} \operatorname{ind}\left(T_{H}\left(g_{i}\right)\right) .
$$

Müller's Theorems (§5.1) handle occurrences when $\hat{X} / H$ has genus 0 with one extra condition; it has at most two places over $z=\infty$. $\S 5.1$ discusses where this condition appears in [Mü196]. There are, however, likely further cases from those he cites where $\hat{X} / H$ has genus 0 . Excluding the cases of degree 5 of this paper, and the Davenport polynomials from [Fri86], his examples are polynomial covers of $\mathbb{P}_{z}^{1}$ with two finite branch points. To complete considering $\mathcal{R}_{f}(K) \backslash \mathcal{V}_{f}(K)$ leaves knowing, when the genus is 0 , if corresponding families have fibers with rational points. This was the concern of [DF90a]. When $\hat{X} / H$ has genus 1 , there is no general technique to decide if these Nielsen classes are arithmetically exceptional.

4.5. Example of a family of genus 1 covers akin to Main Prop. 1.3. As above, use description by branch cycles. Here ${ }_{+} \mathrm{C}_{1}$ and ${ }_{+} \mathrm{C}_{3}$ are the conjugacy classes of 2-cycles in $S_{5},+\mathrm{C}_{2}$ is the conjugacy class of a 3 -cycle and ${ }_{+} \mathrm{C}_{4}$ is the conjugacy class of a 5 -cycle.

The corresponding Nielsen class is ${ }_{+} \mathrm{Ni}_{\infty}=\mathrm{Ni}_{\infty}\left(S_{5},{ }_{+} \mathbf{C}\right)$ :

$$
\left\{\boldsymbol{g}=\left(g_{1}, \ldots, g_{4}\right) \mid g_{1} g_{2} g_{3} g_{4}=1,\langle\boldsymbol{g}\rangle=S_{5} \text { and } \boldsymbol{g} \in{ }_{+} \mathbf{C}\right\} .
$$

As with List A of $\S 1.2$, there is a moduli space $\mathcal{H}\left(S_{5},{ }_{+} \mathbf{C}\right)_{\infty}={ }_{+} \mathcal{H}_{\infty}$ of such polynomials. We compute as in $\S 1.3$ to see ${ }_{+} \mathcal{H}_{\infty}$ is a parameter space for a family of degree 10 covers. Here, however, $+\mathcal{H}_{\infty}$ parameterizes genus 1 covers of $\mathbb{P}_{z}^{1}$. Then, as above, we check that ${ }_{+} \mathcal{H}_{\infty}$ is irreducible and its $\mathbb{Q}$ points are Zariski dense. 
4.5.1. Genus of the corresponding degree 10 covers. Let $\boldsymbol{g}$ be a branch cycle description of the cover from $+\mathrm{Ni}_{\infty}$. Compute the genus $g$ of $+\mathcal{T}_{\boldsymbol{p}}^{(2)}$ from Riemann-Hurwitz:

$$
2(10+g-1)=\sum_{i=1}^{4} \operatorname{ind}\left(R\left(g_{i}\right)\right) .
$$

Suppose $g_{1}$ and $g_{3}$ are 2-cycles from $S_{5}$. Then, $R\left(g_{i}\right)$ has shape $(2)(2)(2)$ in the representation $R, i=1,3$. Similarly, if $g_{2}$ is a 3-cycle, $R\left(g_{3}\right)$ has shape $(3)(3)(3)$. Finally, $R\left(g_{4}\right)$ has shape $(5)(5)$. Thus, the total contribution to the right side of $(4.8)$ is $2 \cdot 3+6+2 \cdot 4=20$ and $g=1$.

Next: Compute ${ }_{+} \mathrm{Ni}_{\infty}$ modulo conjugation by $S_{5}$. Choose $S_{5}$ representatives with $g_{4}$ equal $g_{\infty}=(12345)^{-1}$. Divide ${ }_{+} \mathrm{Ni}_{\infty}$ into two sets $T_{1}$ and $T_{2}$ : $\boldsymbol{g} \in T_{1}$ has $g_{1}$ and $g_{2}$ with no integers of common support, and $\boldsymbol{g} \in T_{2}$ has $g_{1}$ and $g_{2}$ with one integer of common support. Conjugate by a power of $\boldsymbol{g}_{\infty}$ to assure elements of $T_{1}$ have $g_{1}=(1 j)$ with $j=2$ or 3 . Similarly, elements in $T_{2}$ have 1 as common support of $g_{1}$ and $g_{2}$. From this, list ${ }_{+} \mathrm{Ni}_{\infty}{ }^{\text {abs }}$.

4.5.2. List B. ${ }_{+} \mathrm{Ni}_{\infty}{ }^{\text {abs }}$ representatives with 2 nd position a 3 -cycle.
a) $((12),(345),(13))$
b) $((12),(145),(34))$
c) $((12),(135),(45))$
d) $((12),(134),(15))$
e) $((13),(145),(23))$.

Similar lists have the type (3) element in the 1st or in the 3rd positions. Apply braid elements $Q_{1}$ (resp., $Q_{2}$ ) to List B to produce these. Finally: Draw conclusions exactly as in $\S 1.3$. Start by computing the $a_{1, j} \mathrm{~s}$ :

$$
a_{1,2}=Q_{1}^{-2}=\left(0_{b} 0_{e} 0_{d} 0_{c}\right), a_{1,3}=Q_{1} Q_{2}^{-2} Q_{1}^{-1}=\left(0_{a} 0_{d} 0_{e}\right) .
$$

These elements generate $S_{5}$ itself - a transitive group - in their action on List B. As previously, consider a 3rd element's action on List B:

$$
a_{1,4}=Q_{1} Q_{2} Q_{3}^{-2} Q_{2} Q_{1}^{-1}=\left(0_{a} 0_{b} 0_{c} 0_{d}\right) .
$$

Expression (1.3a) shows the product

$$
a_{1,2} a_{1,3} a_{1,4}=\left(Q_{1} Q_{2} Q_{3}^{2} Q_{2} Q_{1}\right)^{-1}
$$

acts trivially on ${ }_{+} \mathrm{Ni}_{\infty}{ }^{\text {abs }}$. Thus, $\left(a_{1,2}, a_{1,3}, a_{1,4}\right)$ are branch cycles for a degree 5 cover $+\varphi:{ }_{+} \mathcal{H}_{\infty 0,1, \infty} \rightarrow \mathbb{P}^{1}$ of the sphere ramifying at 3 -places: $0,1, \infty$. Riemann-Hurwitz gives the genus of this cover as 0 . Apply $\S 6.1$ to see $+\mathcal{H}_{\infty}$ has a dense set of rational places.

Proposition 4.4. The family ${ }_{+} \Psi:{ }_{+} \mathcal{T}^{(2)} \rightarrow{ }_{+} \mathcal{H}_{\infty} \times \mathbb{P}_{z}^{1}$ has a section making it a family of elliptic curves. Suppose $\boldsymbol{p} \in{ }_{+} \mathcal{H}_{\infty}(\mathbb{Q})$ has fiber ${ }_{+} \mathcal{T}_{\boldsymbol{p}}^{(2)}$. Then, $\left.\right|_{+} \Psi_{\boldsymbol{p}}\left({ }_{+} \mathcal{T}_{\boldsymbol{p}}^{(2)}(\mathbb{Q})\right) \cap f_{\boldsymbol{p}}(\mathbb{Q}) \mid<\infty$. 
Proof. Consider any cover $+\varphi^{(2)}:{ }_{+} \mathcal{T}_{\boldsymbol{p}}^{(2)} \rightarrow \mathbb{P}_{z}^{1}$ in the family. The unique unramified point in $+\varphi^{(2)}$ over the branch point of the 3 -cycle has a $\mathbb{Q}(\boldsymbol{p})$ rational point. This point serves as the fiber origin on the family of genus 1 curves.

The last sentence is the analog of Prop. 2.5. This follows if the normalization ${ }_{+} W$ of the fiber product $W=\mathbb{P}_{y}^{1} \times_{\mathbb{P}_{z}^{1}}+\mathcal{T}_{\boldsymbol{p}}^{(2)}$ has but finitely many $\mathbb{Q}$ points lying over $\mathbb{Q}$ values of $z$. Faltings' Theorem gives this if each component of the fiber product has genus at least 2 .

Prop. 2.5's proof shows components of $W$ correspond to $S_{5}(1)$ orbits in the representation $R$. There are two such orbits: $\left\{s_{1}^{\prime}, \ldots, s_{4}^{\prime}\right\}$; and the complement of this set of symbols from $\S 2.2$. Label corresponding components $W_{1}$ and $W_{2}$.

Abhyankar's Lemma $\S 7$ lets us list points from $\mathbb{P}_{y}^{1}$ ramified in $W$ :

(4.9a) The three values $y_{1, j}$ unramified over $z_{1}, k=1,2,3$;

(4.9b) the two points $y_{2, k}, k=1,2$, unramified over $z_{2}$; and

(4.9c) three points $y_{3, l}, l=1,2,3$, unramified over $z_{3}$.

Again, take branch cycles as conjugates, fixing 1 in the standard representation of $S_{5}$, of branch cycles for the corresponding points $z_{i}, i=1,2,3$.

Apply Riemann-Hurwitz. So, $2\left(4+g\left(W_{1}\right)-1\right)=e_{1}+e_{2}+e_{3}$ where $e_{1}$ (resp., $e_{3}$ ), the contribution from $y_{1, k} \mathrm{~s}$ (resp., $y_{3, l} \mathrm{~s}$ ), is 3 and $e_{2}$, from the $y_{2, k} \mathrm{~s}$ is 4 . Thus, $g\left(W_{1}\right)$ is 2 . Similarly, $2\left(6+g\left(W_{2}\right)-1\right)=12+8=20$ or $g\left(W_{2}\right)=5$.

Problem 4.5. Is there a Zariski dense set of $\boldsymbol{p} \in{ }_{+} \mathcal{H}_{\infty}(\mathbb{Q})$ for which ${ }_{+} \mathcal{T}_{\boldsymbol{p}}^{(2)}$ has an infinite set of $\mathbb{Q}$ points.

\section{Müller's additions to [Fri86].}

$\S 5.1$ reviews Müller's (and [Fri86]'s) applications to Hilbert's Irreducibility Theorem of variation of a coefficient, other than the constant term, in a fixed polynomial. Müller goes beyond [Fri86] at two places. He treats variation of the penultimate coefficient (next to the constant term, instead of the constant term). He does this by describing all geometric monodromy groups from covers of the form $f(y) / y$ with $(\S 5.2)$. He also rediscusses over an arbitrary number field the $[\mathbf{F r i 8 6}]$ results for varying the $y^{i}$ coefficient for $2 \leq i \leq n-2$ over $\mathbb{Q}$. [Fri99, $\S 7-\S 8]$ contains much of what we quote from [Fri86], including a complete description of the Davenport polynomials of degree 13, in the style of examples here.

5.1. Variation of coefficients other than the constant term. [Mül96] reviews known results from [Fri86] and [Fri74]. Beyond Explicit HIT Theorem 1.1, a more general result over any number field considers variation of the constant coefficient of a fixed polynomial. Use previous notation, except replace $\mathbb{Q}$ with a general number field $K$. As before, $\mathcal{A}$ is a fractional ideal 
of $K$. (Results still hold if $\mathcal{A}$ is a subring of $K$, finitely generated over $\mathcal{O}_{K}$.) The following is in [Fri86, Th. 7.2].

Result 5.1 (HIT). Suppose $f \in K[y]$ and $\mathcal{R}_{f}(\mathcal{A}) \backslash \mathcal{V}_{f}(\mathcal{A})$ is infinite. If $f$ is indecomposable (over $K$ ) then, either $\operatorname{deg}(f)=5$ or $\operatorname{deg}(f) \in\{7,11,13,15$, $21,31\}$.

The Hurwitz space approach to the degree 5 example allows immediate extension over any number field. So, exceptions to finiteness of $\mathcal{R}_{f}(\mathcal{A}) \backslash \mathcal{V}_{f}(\mathcal{A})$ over any number field $\mathrm{K}$ for each fractional ideal $\mathcal{A}$ come from the degree 5 case. [Fri86] completely discusses exceptional degrees $\{7,11,13,15,21,31\}$. The Hurwitz space analysis for this is more demanding. It was the first foray (from 1968) in practical use of Hurwitz spaces. Yet, the arithmetic analysis is simpler than for $§ 2.3-\S 2.5$. This is because polynomials in these families (of degrees $\{7,11,13,15,21,31\}$ ) have no contribution to $\mathcal{R}_{f}(\mathcal{A})$ from nonpolynomial rational functions. Still, we explain why $m=7$ and $m=13$ are especially interesting.

Let $m$ be an integer from $\{7,11,13,15,21,31\}$. For each $m$ there is a least field of definition $K_{m} \neq \mathbb{Q}$ for polynomials of that degree providing exceptions to HIT Result 5.1. The extension $K_{m} / \mathbb{Q}$ has degree equal the number of conjugacy classes in $N_{S_{m}}(G)$ of $m$-cycles from $G$, the monodromy group of the polynomial. For example, $K_{7}=\mathbb{Q}(\sqrt{-7})$, and $K_{13}=\mathbb{Q}\left(\zeta_{13}+\right.$ $\left.\zeta_{13}^{3}+\zeta_{13}^{9}\right)$, a degree 4 extension of $\mathbb{Q}$. For each $m$ and $m$-cycle conjugacy class, there is one corresponding absolutely irreducible Hurwitz space of polynomials, defined over $K_{m}$. For each case the Hurwitz parameter space is a rational variety. (It was exactly the techniques of this paper that gave this.) This is most compelling for $m=7$ and 13 where exceptions form families of polynomials having three (finite) branch points. In the other cases, the polynomials have only two finite branch points, altogether three branch points, counting $\infty$.

Now we explain the possibilities for variation of other coefficients of a fixed polynomial $f \in K[y]$ with $K$ a number field. Consider the $i$ th coefficient of $f$, with $1 \leq i \leq n-1$. For varying this, define $\mathcal{V}_{f, i}(\mathcal{A})$ to be

$$
\left\{z_{0} \in \mathcal{A} \mid \exists y_{0} \in K \text { with } f\left(y_{0}\right)-z_{0} y_{0}^{i}=0\right\} .
$$

Define $\mathcal{R}_{f, i}(\mathcal{A})$ similarly. Assume the following:

a) $f(0) \neq 0 ;$ b) $\operatorname{gcd}(i, n)=1$; and

c) $2 \leq i \leq n-2$.

[Fri86, Thm. 7.4] contains the following (Müller comments on this in [Mül96, §10]).

Result 5.2 (HIT $i$-Result, $2 \leq i \leq n-2$ ). Assume $i$ and $f$ satisfy (5.1). Then $\mathcal{R}_{f, i}(\mathcal{A}) \backslash \mathcal{V}_{f, i}(\mathcal{A})$ is finite.

Remark 5.3 (Classification Comments). Result 5.1 uses the classification of finite simple groups. This comes by directly or indirectly quoting results 
from [Kan80] that list doubly transitive groups. Still, Result 5.2 uses little more than a combination of the ingredients of Result 5.1 and Marggraf's old theorem (see §5.2). The outcome gives much to a classification of variables separated rational functions $f(x)-g(y)$. Specifically it does classify those where $g$ is either a polynomial or its denominator is a power of a linear or quadric polynomial. When $K=\mathbb{Q}$ and $g$ is a polynomial, the result is in [Fri74] and it is classification free.

5.2. Group theory and the HIT 1-Result. Conditions (5.1a) and (5.1b) allow assuming indecomposability of the rational function $f(y) / y^{i}$. There should be more precise results even removing these conditions. Still, the case $i=1$ (or equivalently, $i=n-1$ ), posed by [Fri86], gives serious new phenomena [Mül96, Th. 1.3].

Result 5.4 (HIT 1-Result). Assume (5.1a). If $K=\mathbb{Q}$ then $\mathcal{R}_{f, 1}$ is finite. If $K \neq \mathbb{Q}$ and $\mathcal{R}_{f, 1}(\mathcal{A}) \backslash \mathcal{V}_{f, 1}(\mathcal{A})$ is infinite, then $n \in\{4,6,8,9,12,16\}$. Further, suppose $n$ is one of these exceptional integers. Then, there are number fields $K$ and $f \in K[y]$ for which $\mathcal{R}_{f, 1}(\mathcal{A}) \backslash \mathcal{V}_{f, 1}(\mathcal{A})$ is infinite for some fractional ideals $\mathcal{A}$.

As above, the classification of finite simple groups appears when using a list of 2-transitive groups of degree $n$. [Mül96] proceeds by listing those containing an $(n-1)$-cycle. Recall the group theory statements that appear in Comments on Prop. 4.2. [Mül96] lists geometric monodromy groups of rational functions $f(y) / y$ with $f \in K[y]$ and $\mathcal{R}_{f, i}(\mathcal{A}) \backslash \mathcal{V}_{f, i}(\mathcal{A})$ infinite for some fractional ideal $\mathcal{A}$. The remainder of the section describes the exact conditions on a group $G$ for there to exist such a triple $(f, K, \mathcal{A})$. Analogous to $\S 4.4$, a necessary condition is that $G$ have a subgroup $H$ for which $\hat{X} / H$ has genus 0 , producing a rational function $h_{f}$ (below).

5.2.1. [Mül96] starting assumptions. Since $G$ is doubly transitive, its minimal normal subgroup is a simple (nonabelian) group or it is abelian and we take it to be $A=\mathbb{F}_{p}^{m}$ (the affine case). In the affine case, $G$ is transitive on $A$ (since $A$ is transitive on $A$ ). So, $G$ is isomorphic to $\mathbb{F}_{p}^{m} \times{ }^{s} U$ with $U$ a subgroup of $\mathrm{GL}_{m}\left(\mathbb{F}_{p}\right)$ acting faithfully on $A$.

5.2.2. Quotation of [Kan80]. [Kan80, Lemma 6.3] assumes $G$ is an affine group of degree $n$ containing an $n$-1-cycle. Then, $n=q^{e}$ for some prime power $q$ and $G=\mathbb{F}_{q}^{e} \times{ }^{s} U$ with $\operatorname{GL}_{e}(q) \leq U \leq \Gamma L_{e}(q)$. Interpret the singer cycle as a generator of the multiplicative group of nonzero elements of $\mathbb{F}_{q^{e}} \cong \mathbb{F}_{q}^{e}$ (as a vector space over $\mathbb{F}_{q}$ ). This gives an $n$-1-cycle in identifying the integers of the representation with $\mathbb{F}_{q}^{e}$.

5.2.3. [Mül96] Main Theorem. Call a group projective if $\operatorname{PSL}_{m}(q) \leq$ $G \leq P \Gamma L_{m}(q)$ where $G$ acts on points of the projective space of dimension $m-1$. Let $M_{n}, n \in\{11,12,22,23,24\}$ be the Mathieu groups. (The most 
interesting case in [Mül96] is $M_{11}$, with its 3-transitive representation of degree 12.) The next theorem requires the classification of finite simple groups. Without it, [Mül96, Th. 6.1] proves only that $G$ is affine, or $\operatorname{PSL}_{2}(p)$ with $p \geq 5$, or 3 -transitive.

Theorem 5.5. If $G$ is transitive and contains an $n-1$-cycle, then $G$ is one of the following: Affine, $A_{n}$ ( $n$ even), $S_{n}, \mathrm{PSL}_{2}(p)$ or $\mathrm{PGL}_{2}(p)$ with $p \geq 5$, $M_{11}$ (of degree 12 ), $M_{12}$ or $M_{24}$.

[Mü196, Prop. 7.1] describes those from this list having an intransitive subgroup $V$ (choose it maximal among intransitive subgroups) and

(5.2) an $n$-1-cycle $g$ having at most two orbits on the coset space $G / V$. As [Fri86] and [Mül96, Prop. 4.1 (4)] note, the representation on $G / V$ is automatically faithful. Given $f$, condition (5.2) translates to $h_{f}$ having exactly two points over $z=\infty$.

5.2.4. Fractional values. Statement (5.2) is necessary (from Siegel's Theorem over general number fields) for $h_{f}$ to have infinitely many values in some fractional ideal. [Fri86, Thm. 7.2] (and [Mül96, Prop. 5.2]) also note the following: Orbits of $g$ in (5.2) have lengths $k$ and $\ell$ where $\operatorname{lcm}(k, \ell)=n-1$ and $k+\ell \geq n$. The latter condition follows from a 2-transitive group having no intransitive coset representation of degree smaller than $n$. Further, if the degree is $n$, and the subgroup is intransitive, the (group) characters of the two permutation representations are the same. From this conclude $k=n-1$ and $\ell \mid n-1$.

Further, even in excluding the case $2 \leq i \leq n-2$, [Mü196, Prop. 4.2] and [Fri86] use the two-orbit condition. This occurs in applying Marggraf's Theorem [Wie64, p. 13.8] to $g^{n-i}$ being an $i$-cycle and concluding $G=A_{n}$ or $S_{n}$. The following result is a [Mül96, Prop. 7.1] conclusion.

Theorem 5.6. Cycle lengths of $g$ on $G / V$ are $n-1$ and $\ell$ with $G$ in the following.

(5.3a) $G=A \times{ }^{s} U$ with $A \equiv \mathbb{F}_{p}^{m}, U \leq G L_{m}(p)$ and $n=p^{m}$. With $p^{r}$ the index of $V \cap A$ in $A, \ell=\left(p^{m}-1\right) /\left(p^{r}-1\right)$. Let $V^{\prime}$ be a conjugate of $V$ with $\left|V^{\prime} \cap U\right|$ maximal. Then $V^{\prime}=N_{U}\left(V^{\prime} \cap A\right)\left(V^{\prime} \cap A\right)$.

(5.3b) If $G$ is not affine, then either $G$ is a group from Thm. 5.5 ( $V$ is a point stabilizer) or $G$ is among the following:

- $n=6, \ell=5, G=\mathrm{PSL}_{2}(5)$ and $V$ is isomorphic to $D_{3}$;

- $n=8, \ell=7, G=\mathrm{PSL}_{2}(7)$; and $V=A_{4}$; or

- $n=12, \ell=11, G=M_{11}$ and $V=A_{6}$.

[Mül96, Prop. 7.2] shows the affine cases produce examples satisfying all the hypotheses of [Mü196, Th. 6.2] and (5.2), the two-orbit condition. The genus 0 condition, from applying Riemann-Hurwitz to generators of $G$ for both representations, excludes all but finitely many examples. For example, [Mü196] uses [GT90] and [Neu93] to handle the affine case. In particular, 
with (5.2) in force, [Mül96, Lemma 8.4] discusses how a GAP program cuts the list of possibilities - details from Müller's thesis. Suppose we drop (5.2) while retaining that a branch cycle description for the representation of $G$ on $G / V$ gives genus 0 or 1 . This would likely produce more exceptional examples like those of $\S 4.4$.

\section{Rational point criterion for Hurwitz spaces.}

We give a practical if and only if criterion for Hurwitz spaces of four branchpoint covers to be birational images of some projective space. This $\mathbb{Q}$ unirationality test often shows when spaces like $\mathcal{H}_{\infty}$ have a dense set of rational points. The test depends only on braid group action. This phrasing is for full Hurwitz families: Parameterizing all covers in a given Nielsen class $\operatorname{Ni}(G, \mathbf{C})$, with $\mathbf{C}$ consisting of $r \geq 4$ conjugacy classes of $G$ and $G$ a transitive subgroup of $S_{n}$.

The test for $r=4$ of Prop. 6.5 is the best, most likely and easiest to apply criterion. For $r=4$, the $\mathrm{SL}_{2}(\mathbb{C})$ quotient action presents each Hurwitz space component as a canonical cover of the classical $j$-line. The immediate application to $\mathcal{H}_{\infty}$ follows from $\S 1.1$. The criterion computes a branch cycle description for this cover. The test is that the genus computed from this is 0 . Though it is less likely, when the test of $\S 6.1$ holds there are extra diophantine corollaries. In particular, it implies the Hurwitz space with data for the branch points of the covers in the family is unirational. Further, we need the computation that gives us this in $\S 6.4 .3$ for the proof of Prop. 6.5.

6.1. Tesselating a Hurwitz space with curves. Denote $\mathbb{P}^{r} \backslash D_{r}$ by $U_{r}$. Hurwitz monodromy action on $\mathrm{Ni}(G, \mathbf{C}) / N_{S_{n}}(G)=\mathrm{Ni}(G, \mathbf{C})^{\text {abs }}$ produces an unramified cover $\Psi_{G, \mathbf{C}}: \mathcal{H}(G, \mathbf{C}) \rightarrow U_{r}(\S 1.2)$. As in $\S 1.3$, pull this cover back to $U^{r}=\left(\mathbb{P}^{1}\right)^{r} \backslash \Delta_{r}$ by forming the fiber product $\mathcal{H}(G, \mathbf{C})^{\prime}=$ $\mathcal{H}(G, \mathbf{C}) \times_{U_{r}} U^{r}$. This may have several components as [DF90a] takes pains to explain. These components correspond exactly to orbits of the straight braid group (kernel of the natural map of the braid group to $S_{r}$ ) acting on the straight Nielsen class $\operatorname{SNi}(G, \mathbf{C})$. Define straight Nielsen classes exactly as Nielsen class $(\S 1.1)$, except $r$-tuples $\boldsymbol{g}=\left(g_{1}, \ldots, g_{r}\right)$ have entries in $\mathbf{C}$ in a fixed order: $g_{i}$ is in the conjugacy class $\mathrm{C}_{i}, i=1, \ldots, r$. Example: There are (at least) $r$ ! components if all conjugacy classes in $\mathbf{C}$ are distinct when extended to conjugacy classes in $N_{S_{n}}(G)$. Then, each component is a copy of a component of $\mathcal{H}(G, \mathbf{C})$; an especially easy case.

Even if $\mathcal{H}(G, \mathbf{C})$ is absolutely irreducible with $\mathbb{Q}$ as field of definition, absolutely irreducible components of $\mathcal{H}(G, \mathbf{C})^{\prime}$ may not have field of definition $\mathbb{Q}$. For example, suppose $\mathbf{C}$ satisfies the following.

(6.1a) $\mathbf{C}$ is a rational union of conjugacy classes, and

(6.1b) a conjugacy class (say $\mathrm{C}_{1}$ ) is not a rational conjugacy class even in its extension to $N_{S_{n}}(G)$. 
Now, assume $\sigma \in G_{\mathbb{Q}}$ maps to $k \in G\left(\mathbb{Q}^{\text {cyc }} / \mathbb{Q}\right)$ and has the following properties.

(6.2a) $k$ is relatively prime to the order of the elements of $\mathrm{C}_{1}$.

(6.2b) The extension of conjugacy classes $\mathrm{C}_{1}^{k}$ and $\mathrm{C}_{1}$ in $N_{S_{n}}(G)$ aren't equal.

Then, $\sigma$ acts nontrivially on the absolutely irreducible components of $\mathcal{H}(G, \mathbf{C})^{\prime}$. This is a consequence of the branch cycle argument in [Fri95a, $\S 3]$ or $[$ Fri77, §5]. The goal of this section is to give a criterion for $\mathcal{H}(G, \mathbf{C})$ having a $\mathbb{Q}$ point. For this we need an extra hypothesis. As in $\S 1.3$, consider these elements of $S H_{r}$ :

$$
\begin{aligned}
a_{1,2}=Q_{1}^{-2}, a_{1,3} & =Q_{1} Q_{2}^{-2} Q_{1}^{-1}, a_{1,4}=Q_{1} Q_{2} Q_{3}^{-2} Q_{2}^{-1} Q_{1}^{-1}, \ldots, \\
a_{1, r} & =Q_{1} \cdots Q_{r-2} Q_{r-1}^{-2} Q_{r-2}^{-1} \cdots Q_{1}^{-1} .
\end{aligned}
$$

These elements have indices in their action on $\operatorname{SNi}(G, \mathbf{C})$. The following hypothesis appears in Prop. 6.2.

(6.3) $\mathcal{H}(G, \mathbf{C})^{\prime}$ has an absolutely irreducible $\mathbb{Q}$-component.

Here is a criterion for this [Fri77, Thm. 5.1].

Proposition 6.1. Hypothesis (6.3) holds if the following holds.

(6.4a) $S H_{r}$ is transitive on $\operatorname{SNi}(G, \mathbf{C})$, and

(6.4b) the extension of each conjugacy class $\mathrm{C}_{i}$ to $N_{S_{n}}(G)$ is rational in $N_{S_{n}}(G)$.

These hypotheses hold in the cases of this paper. That is, we explicitly display the (6.4a) transitivity in List A and List B. The group $N_{S_{n}}(G)$ here is $S_{n}(n=5)$, a group with all conjugacy classes rational. Here is a general statement.

Proposition 6.2. Assume (6.3) holds and $O^{\prime} \subset \operatorname{SNi}(G, \mathbf{C}) / S N_{S_{n}}(G)$ is an $S H_{r}$ orbit. Let $\mathcal{H}_{O^{\prime}}$ be the absolutely irreducible $\mathbb{Q}$-component of $\mathcal{H}(G, \mathbf{C})^{\prime}$ corresponding to $O^{\prime}$. Suppose $\left|O^{\prime}\right|=N$ and $\left\langle a_{1,2}, \ldots, a_{1, r}\right\rangle$ is transitive on $O^{\prime}$ (automatic when $r=4\left[\mathbf{B F 8 2}\right.$, Lemma 1.6]). Compute $g_{O^{\prime}}$ from

$$
2\left(N+g_{O^{\prime}}-1\right)=\sum_{i=2}^{r} \operatorname{ind}\left(a_{1, i}\right) .
$$

Then, $g_{O^{\prime}}$ is the genus of the nonsingular completion $\overline{\mathcal{H}}_{O^{\prime}, L}$ of $\mathcal{H}_{O^{\prime}, L}$, the restriction of $\mathcal{H}_{O^{\prime}}$ over $L=\left\{\left(z, z_{2}^{0}, \ldots, z_{r}^{0}\right) \mid z \in \mathbb{P}^{1}\right\} \subset U^{r}$. Suppose the following:

(6.6a) $z_{2}^{0}, \ldots, z_{r}^{0}$ are distinct $\mathbb{Q}$ points in $\mathbb{P}_{x}^{1}$.

(6.6b) $g_{O^{\prime}}=0$ and $\overline{\mathcal{H}}_{O^{\prime}, L}$ has a $\mathbb{Q}$ point.

Then, $\mathcal{H}_{O^{\prime}}$ is unirational and has a dense set of rational points. 
Proof. Identify the line $L$ with $\mathbb{P}_{z}^{1} \backslash\left\{z_{2}^{0}, \ldots, z_{r}^{0}\right\}$. [BF82, (4.5) and following discussion] identifies $a_{1,2}, \ldots, a_{1, r}$ with generators of the fundamental group of $L$ modulo the relation $a_{1,2} \cdots a_{1, r}=1$ in $H_{r}$. Further, by restriction of $\mathcal{H}_{O^{\prime}}$ over $L$, the corresponding permutation representation is restriction from $S H_{r}$ acting on $O^{\prime}$. Thus, formula (6.5) is just Riemann-Hurwitz for the cover $\overline{\mathcal{H}}_{O^{\prime}, L} \rightarrow \mathbb{P}^{1}$ computed from the action of branch cycles.

Finally, suppose $z_{2}^{0}, \ldots, z_{r}^{0}$ are distinct $\mathbb{Q}$ points. Then $\overline{\mathcal{H}}_{O^{\prime}, L} \rightarrow \mathbb{P}_{z}^{1}$ is a $\mathbb{Q}$ cover, and $\mathcal{H}_{O^{\prime}}$ is birational to $W=\overline{\mathcal{H}}_{O^{\prime}, L} \times\left(\mathbb{P}^{1}\right)^{r-1}$. Clearly $W$ is unirational (over $\mathbb{Q}$ ) if and only if $\overline{\mathcal{H}}_{O^{\prime}, L}=X$ is unirational. Since $X$ is a nonsingular curve, this is equivalent to $\overline{\mathcal{H}}_{O^{\prime}, L}$ being of genus 0 and having a $\mathbb{Q}$ point.

6.2. $\mathrm{SL}_{2}(\mathbb{C})$ action on Hurwitz spaces and covers of $\Lambda_{r}$ and $J_{r}$. Much of [DF90a] is on criteria guaranteeing rational points on families of genus 0 curves. In particular, the results there apply to consider how to satisfy (6.6b). If $N$ is odd, then $\overline{\mathcal{H}}_{O^{\prime}, L}$ has a rational point. That is the easiest criterion when it holds, and it does in the main cases of this paper. The most refined criterion comes from the action of $H_{r}$ on pointed Nielsen classes as in [DF90a]. We won't review that. Rather, when $r=4$, Prop. 6.5 gives the best practical criterion for a component of $\mathcal{H}(G, \mathbf{C})$ to be unirational. This was the criterion, with fewer details, [Fri90] applied to degree 5 covers of $\mathbb{P}^{1}$ with four conjugacy classes of 3-cycles.

The group $S_{r}$ acts on the space $\left(\mathbb{P}^{1}\right)^{r}$ by permutation of its coordinates. As with action of $B_{r}$, put this action on the right. This gives a natural map $\Psi_{r}:\left(\mathbb{P}^{1}\right)^{r} \rightarrow \mathbb{P}^{r}$. Consider $\boldsymbol{z}=\left(z_{1}, \ldots, z_{r}\right)$ with none of the coordinates equal $\infty$. The image of $z$ under $\Psi_{r}$ has as representative in projective space the point whose coordinates are the coefficients of the polynomial $\prod_{i=1}^{r}\left(x-z_{i}\right)$ in $z$. (If $z_{i}=\infty$, replace the factor $x-z_{i}$ by 1 .) Also, $\Psi_{r}$ takes the fat diagonal $\Delta_{r}$ to $D_{r}$. This interprets $U_{r}=\mathbb{P}^{r} \backslash D_{r}$ as the space of $r$ distinct unordered points in $\mathbb{P}^{1}$. Thus, $\Psi_{r}: U^{r} \rightarrow U_{r}$ is an unramified Galois cover with group $S_{r}$.

Consider $\mathrm{PSL}_{2}(\mathbb{C})$ as linear fractional transformations acting diagonally on $r$ copies of $\mathbb{P}_{z}^{1}$. For $\alpha \in \mathrm{PSL}_{2}(\mathbb{C})$ and $\boldsymbol{z} \in U^{r}, \alpha(\boldsymbol{z}) \mapsto\left(\alpha\left(z_{1}\right), \ldots, \alpha\left(z_{r}\right)\right)$. Put the action of $\mathrm{PSL}_{2}(\mathbb{C})$ on the left, since it commutes with the coordinate permutation action of $S_{r}$. The quotient $\mathrm{PSL}_{2}(\mathbb{C}) \backslash U^{r}=\Lambda_{r}$ generalizes the $\lambda$-line minus the points $0,1, \infty$ from the theory of modular curves. Also, $\mathrm{PSL}_{2}(\mathbb{C}) \backslash U_{r}=J_{r}$ generalizes the $j$-line minus the point at $\infty$. It is a space of complex dimension $r-3$. Take $r=4$. Then, four unordered distinct points correspond to the unique unramified degree two cover of $\mathbb{P}^{1}$ ramified at these four points. This then corresponds to the classical $j$-invariant of the associated elliptic curve. In summary we have the following.

Proposition 6.3 $\left(J_{4}\right)$. The quotient of the (unramified) cover $\Psi_{4}: U^{4} \rightarrow$ $U_{4}$ by $\mathrm{PSL}_{2}(\mathbb{C})$ produces the classical (ramified) map $\psi: \mathbb{P}_{\lambda}^{1} \backslash\{0,1, \infty\} \rightarrow$ 
$\mathbb{P}_{j}^{1} \backslash\{\infty\}$. Though $\psi$ is a Galois cover with group $S_{3}$, this copy of $S_{3}$ naturally identifies with the quotient of $S_{4}$ by the Klein 4-group, rather than with the stabilizer of 1 in $S_{4}$ appearing above.

Proof. Consider $z \in \mathbb{P}^{4} \backslash D_{4}$. Then, $z$ corresponds uniquely to a degree 2 cover $Y \rightarrow \mathbb{P}^{1}$ with associated branch locus $z$. By the Riemann-Hurwitz formula, the genus of $Y$ is one. Thus, regard $\mathbb{P}^{4} \backslash D_{4}$ as a Hurwitz space $\mathcal{H}$, and consider the $\mathrm{PSL}_{2}(\mathbb{C})$ action from $\S 1.1$ on it. Two points $\boldsymbol{z}, \boldsymbol{z}^{\prime} \in \mathbb{P}^{4} \backslash D_{4}$ give genus one covers $\varphi: E \rightarrow \mathbb{P}_{z}^{1}$ (ramified at $\boldsymbol{z}$ ) and $\varphi^{\prime}: E^{\prime} \rightarrow \mathbb{P}_{z}^{1}$ (ramified at $\left.\boldsymbol{z}^{\prime}\right)$. Then, $E$ and $E^{\prime}$ are isomorphic if and only if there exists $\alpha \in \mathrm{PSL}_{2}(\mathbb{C})$ for which the following statement holds:

(6.7) $\alpha(\boldsymbol{z})=\boldsymbol{z}^{\prime}$, or equivalently $\alpha \circ \varphi=\varphi^{\prime}$.

Therefore, $J_{4}$ is the $j$-line, missing only the point at $\infty$; this doesn't correspond to a genus one curve.

We extend the $\mathrm{PSL}_{2}(\mathbb{C})$ action of $\mathbb{P}^{r} \backslash D_{r}$ to Hurwitz spaces. Suppose $\mathcal{H}$ is an absolute (or inner) Hurwitz (moduli) space of $r$-branch point covers of $\mathbb{P}_{x}^{1}$ in a given absolute (or inner) Nielsen class $\mathrm{Ni}(G, \mathbf{C})^{\text {abs }}\left(\right.$ or $\left.\operatorname{Ni}(G, \mathbf{C})^{\text {in }}\right)$. We explain further.

There is a map $\beta_{r}: \mathcal{H} \rightarrow U_{r}$ : If $\boldsymbol{p} \in \mathcal{H}$ has a representative cover $\varphi_{\boldsymbol{p}}$ : $X_{\boldsymbol{p}} \rightarrow \mathbb{P}_{z}^{1}$, then the collection of branch points $z$ of $\varphi_{\boldsymbol{p}}$ determines a point in $U_{r}$. Call this the branch point map. To compute the Nielsen class of the cover choose a standard set of continuous, piece-wise differentiable paths $\mathcal{P}_{1}, \ldots, \mathcal{P}_{r}$ in $\mathbb{P}_{z}^{1} \backslash \boldsymbol{z}$ based at a point $z_{0}$ (not in $\boldsymbol{z}$ ). (For details see [Fri77], [MM], [Ser92] or [Völ96].) The salient points are as follows. For some ordering $\left(z_{1}, \ldots, z_{r}\right)$ of the entries of $\boldsymbol{z}$ :

(6.8a) Each $\mathcal{P}_{i}=\gamma_{i} \delta_{i} \gamma_{i}^{-1}$, with $\delta_{i}$ a clockwise path around $z_{i}$ bounding a closed disc $D_{i}$ with center $z_{i}$;

(6.8b) the $\gamma_{i}$ s meet only at $z_{0}$;

(6.8c) $\gamma_{i}$ meets $D_{i}$ only at the beginning point of $\delta_{i}$, and it doesn't meet $D_{j}$ if $j \neq i$; and

(6.8d) for a suitably small disk $D$ around $z_{0}$, the initial meeting point $m_{i}$ of $\gamma_{i}$ with the boundary of $D$ appear in the clockwise order $m_{1}, \ldots, m_{r}$.

For any element $\alpha \in \mathrm{PSL}_{2}(\mathbb{C})$, compose $\varphi_{\boldsymbol{p}}$ with $\alpha$ to give

$$
\alpha \circ \varphi_{\boldsymbol{p}}: X_{\boldsymbol{p}} \rightarrow \mathbb{P}^{1} \text {. }
$$

As $\alpha$ is conformal, you get paths for computing the Nielsen class for $\alpha \circ \varphi_{\boldsymbol{p}}$ from $\alpha^{-1}\left(\mathcal{P}_{1}\right), \ldots, \alpha^{-1}\left(\mathcal{P}_{r}\right)$ based at $\alpha^{-1}\left(x_{0}\right)$. These paths go around the respective branch points $\alpha^{-1}\left(x_{1}\right), \ldots, \alpha\left(x_{r}\right)$. From this, the cover $\alpha \circ \varphi_{\boldsymbol{p}}$ is in the same Nielsen class as is $\varphi_{\boldsymbol{p}}$. Further, suppose $\varphi_{\boldsymbol{p}}$ is a Galois cover and $\mu_{\boldsymbol{p}}$ is a fixed isomorphism between $G\left(X_{\boldsymbol{p}} / \mathbb{P}_{x}^{1}\right)$ and $G$. So composing $\varphi_{\boldsymbol{p}}$ with $\alpha$ doesn't affect the isomorphism $\mu_{\boldsymbol{p}}$. Thus, $\alpha \in \mathrm{PSL}_{2}(\mathbb{C})$ induces an isomorphism on both absolute and inner Hurwitz spaces, commuting with 
the branch point map $\beta_{r}$. Further, $\mathrm{PSL}_{2}(\mathbb{C})$ is a connected space (containing the identity map). So, any $\alpha$ maps each absolutely irreducible component of $\mathcal{H}$ into itself.

Further, for any Hurwitz space $\mathcal{H}$, consider the space $\mathcal{H}^{\prime}$ of pairs $\left(\boldsymbol{p},\left(z_{1}, \ldots\right.\right.$, $\left.z_{r}\right)$ ) with $\boldsymbol{p} \in \mathcal{H}$ and an ordering $\left(z_{1}, \ldots, z_{r}\right)$ of $\beta_{r}(\boldsymbol{p})=\boldsymbol{z}$. The space $\mathcal{H}^{\prime}$ is the fiber product $\mathcal{H} \times_{U_{r}} U^{r}$. Denote the projection of $\mathcal{H}^{\prime} \rightarrow \mathcal{H}$ off the first factor by $\mathrm{pr}_{1}$. Elements of $\mathrm{PSL}_{2}(\mathbb{C})$ map components of $\mathcal{H}^{\prime}$ isomorphically onto themselves. Finally, both $\mathcal{H}$ and $\mathcal{H}^{\prime}$ are finite covers of the affine space $U_{r}$. Thus, they are affine algebraic varieties. In particular, applying the Reynolds operator argument of $\left[\mathbf{M F} 80\right.$, Th. 1.1] shows the $\mathrm{PSL}_{2}(\mathbb{C})$ quotients of these varieties are also affine varieties. Conclude the next result about the following strong equivalence relation between covers.

(6.9) $\varphi_{i}: X_{i} \rightarrow \mathbb{P}^{1}, i=1,2$, are equivalent if there exists $\alpha: X_{1} \rightarrow X_{2}$ and $\beta: \mathbb{P}^{1} \rightarrow \mathbb{P}^{1}$ with $\varphi_{2} \circ \alpha=\beta \circ \varphi_{1}$.

Proposition 6.4 $\left(\mathrm{PSL}_{2}(\mathbb{C})\right.$-quotient). The quotient spaces $\mathcal{H} / \mathrm{PSL}_{2}(\mathbb{C})=$ $\mathcal{H}^{\text {rd }}$ and $\mathcal{H}^{\prime} / \mathrm{PSL}_{2}(\mathbb{C})$ exist as moduli spaces for the equivalence (6.9) of covers in a Nielsen class. Further, they are affine algebraic varieties and there is a natural commutative diagram of induced maps:

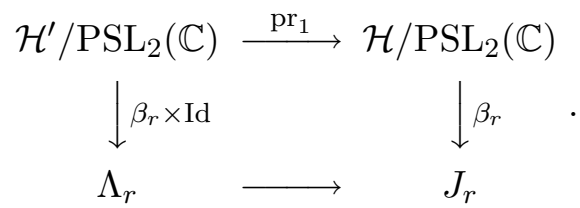

6.3. $\mathrm{SL}_{2}(\mathbb{C})$ action $j$-line covers when $r=4$. Let $\mathcal{H}_{O^{\prime}}$ correspond to an $H_{4}$ orbit $O^{\prime}$ in its action on $\mathcal{H}(G, \mathbf{C})^{\text {abs }}$ (resp. on $\mathcal{H}(G, \mathbf{C})^{\text {in }}$ ). Thus, $\mathcal{H}_{O^{\prime}}$ is an absolutely irreducible component of a Hurwitz space $\mathcal{H}(G, \mathbf{C})$ (over some number field $K$ ) of equivalence classes of $r=4$ branch point covers. Then, Prop. 6.4 produces a finite cover $\beta_{4}^{r d}\left(O^{\prime}\right): \mathcal{H}_{O^{\prime}} / P S L_{2}(\mathbb{C})=\mathcal{H}_{O^{\prime}}^{r d} \rightarrow \mathbb{P}_{j}^{1} \backslash\{\infty\}$. Complete this to a cover $\bar{\beta}^{r d}\left(O^{\prime}\right): \overline{\mathcal{H}}_{O^{\prime}}^{r d} \rightarrow \mathbb{P}_{z}^{1}$.

Let $q_{1}, q_{2}, q_{3}$ be the images of $Q_{1}, Q_{2}, Q_{3}$ in the mapping class group $M_{4}$ (1.3b). Form one further equivalence on $\mathrm{Ni}(G, \mathbf{C})^{\text {abs }}$ (or on $\operatorname{Ni}(G, \mathbf{C})^{\text {in }}$ ). Recall: For $\boldsymbol{g} \in \mathrm{Ni}(G, \mathbf{C}), g_{1} g_{2} g_{3} g_{4}=1$. For $\boldsymbol{g} \in \mathrm{Ni}(G, \mathbf{C}),\left(Q_{1} Q_{3}^{-1}\right)^{2}$ has this effect:

$$
\boldsymbol{g} \mapsto\left(g_{1} g_{2} g_{1} g_{2}^{-1} g_{1}^{-1}, g_{1} g_{2} g_{1}^{-1}, g_{4}^{-1} g_{3} g_{4}, g_{4}^{-1} g_{3}^{-1} g_{4} g_{3} g_{4}\right) \equiv^{G} \boldsymbol{g} .
$$

Form $\operatorname{Ni}(G, \mathbf{C}) /\left\langle Q_{1} Q_{3}^{-1}\right\rangle$. Denote this $\operatorname{MQ}(G, \mathbf{C})$ for mapping quotient classes. Action of $H_{4}$ on $\mathrm{Ni}(G, \mathbf{C})^{\text {abs }}$ induces action of $H_{4} /\left\langle Q_{1} Q_{3}^{-1}\right\rangle=\bar{H}_{4}$ on $\operatorname{MQ}(G, \mathbf{C})$. As for $\operatorname{Ni}(G, \mathbf{C})^{\text {abs }}$ (resp. $\left.\operatorname{Ni}(G, \mathbf{C})^{\text {in }}\right)$ there is the quotient set $\operatorname{MQ}(G, \mathbf{C})^{\text {abs }}$ (resp. $\left.\mathrm{MQ}(G, \mathbf{C})^{\mathrm{in}}\right)$. The next result computes branch cycles for the cover $\bar{\beta}^{r d}\left(O^{\prime}\right)$.

Proposition 6.5 ( $j$-Line Branch Cycles). The group $\bar{H}_{4}$ has generators $\alpha$ $=q_{1} q_{2}$ and $\gamma=q_{1} q_{2} q_{1}$ subject to the relations $\alpha^{3}=\gamma^{2}=1: \bar{H}_{4} \equiv \mathrm{PSL}_{2}(\mathbb{Z})$. 
Orbits of $\bar{H}_{4}$ on $\mathrm{MQ}(G, \mathbf{C})^{\text {abs }}$ (resp. $\mathrm{MQ}(G, \mathbf{C})^{\mathrm{in}}$ ) correspond one-one to orbits of $\mathrm{H}_{4}$ on $\mathrm{Ni}(G, \mathbf{C})^{\text {abs }}$ (resp. $\left.\mathrm{Ni}(G, \mathbf{C})^{\mathrm{in}}\right)$. In particular, consider the orbit $O^{\prime}$ in the discussion before this proposition. Let $\alpha^{\prime}$ and $\gamma^{\prime}$ be respective actions of $\alpha$ and $\gamma$ on the image of $O^{\prime}$ in $\operatorname{MQ}(G, \mathbf{C})^{\text {abs }}$. Then $\left(\alpha^{\prime}, \gamma^{\prime}, q_{2}^{\prime}\right)$ is a description of the branch cycles of the cover $\bar{\beta}^{r d}\left(O^{\prime}\right)$.

Finally, $\mathcal{H}_{O^{\prime}}$ is unirational over $K$ if and only if the genus of $\overline{\mathcal{H}}_{O^{\prime}}^{\text {rd }}$ is 0 and

(6.12) there is a $K$ rational divisor of odd degree on $\overline{\mathcal{H}}_{O^{\prime}}^{r d}$.

$\S 6.4$ completes the proof of Prop. 6.5. This subsection concludes with comments on practical use of (6.12).

Remark 6.6. Testing (6.12). The cover $\bar{\beta}^{r d}\left(O^{\prime}\right): \overline{\mathcal{H}}_{O^{\prime}}^{r d} \rightarrow \mathbb{P}_{j}^{1}$ has branch points 0,1 and $\infty$. If $\bar{\beta}^{r d}\left(O^{\prime}\right)$ has odd degree, then a general $K$ fiber of the cover has odd degree. Often, however, we can do better than that.

Suppose $\varphi: X \rightarrow \mathbb{P}_{z}^{1}$ is any cover with a branch point $z^{\prime}$ in $K$. Then the disjoint cycles of the branch cycle $g_{z^{\prime}}$ provide one quick test for a $K$ rational divisor of odd degree. Let $e$ be an integer for which $g_{z^{\prime}}$ has a disjoint cycle of length $e$. Let $n_{e}$ be the number of disjoint cycles of $g_{z^{\prime}}$ of length $e$. Then, the points of the fiber $X_{z^{\prime}}$ ramified of index $e$ over $z^{\prime}$ correspond to the respective disjoint cycles of length $e$. These form a $K$ rational divisor on $\overline{\mathcal{H}}_{O^{\prime}}^{r d}$. Reason: The action of $G_{K}$ on the fiber over $z^{\prime}$ permutes the points of ramification $e$ among themselves, so it leaves this set of points invariant.

6.4. Proof of Prop. 6.5. Consider the group $\bar{H}_{4}$ of $\S 6.3$. The first subsection presents this group by generators and relations. Denote the element of $\mathrm{PSL}_{2}(\mathbb{C})$ taking $z$ to $1 / z$ by $\mu$. The second subsection shows how quotient action of $\mathcal{H}^{\text {abs }}$ by $\mu$ interprets that the cover $\bar{\beta}^{r d}\left(O^{\prime}\right): \overline{\mathcal{H}}_{O^{\prime}}^{r d} \rightarrow \mathbb{P}_{j}^{1}$ has monodromy group a quotient of $\bar{H}_{4}$. The remainder of the proof follows quickly from these observations.

6.4.1. Presentation of $\bar{H}_{4}$. Relations (1.3a) and $Q_{1} Q_{3}^{-1}$ show that $q_{1} q_{2} q_{1} q_{1} q_{2} q_{1}$ in $\bar{H}_{4}$. From $Q_{1} Q_{2} Q_{1}=Q_{2} Q_{1} Q_{2}$ in $H_{4}$,

$$
q_{1} q_{2} q_{1} q_{1} q_{2} q_{1}=\left(q_{1} q_{2}\right)^{3}=\left(q_{1} q_{2} q_{1}\right)^{2} .
$$

This shows the identities $\alpha^{3}=\gamma^{2}=\alpha \gamma q_{2}$ from Prop. 6.5. All act trivially on $\operatorname{MQ}(G, \mathbf{C})^{\text {in }}$, since they equal the action of $Q_{1} Q_{2} Q_{3} Q_{3} Q_{2} Q_{1}$. So, $\alpha^{\prime} \gamma^{\prime} q_{2}^{\prime}$ is the identity. It is a candidate for a branch cycle description.

6.4.2. The effect of $\mu$ on $\mathcal{H}^{\text {in }}$ or $\mathcal{H}^{\text {abs }}$. First consider the effect of $\mathrm{PSL}_{2}(\mathbb{C})$ on $\mathcal{H}^{\text {in }}$ (or similarly, on $\mathcal{H}^{\text {abs }}$ ). For this argument, refer to either of these choices as $\mathcal{H}$. Suppose $\boldsymbol{p} \in \mathcal{H}$ lies over $\boldsymbol{z}=\left\{z_{1}, z_{2}, z_{3}, z_{4}\right\} \in U_{4}$. Then, any other point $\boldsymbol{p}^{\prime} \in \mathcal{H}$ to another point $\boldsymbol{p}^{\prime \prime} \in \mathcal{H}$ lying over $\boldsymbol{z}$. See the comments of $\S 6.5$ for the fiber product formulation of this. Prop. 6.8 reminds of an 
explicit formula for the $j$-invariant of the genus one curve ramified over the $z$-line at $z$. If $z_{4}=\infty$, then [Rob73, p. I.61] gives the classical formula:

$$
j=-4\left(z_{1} z_{2}+z_{2} z_{3}+z_{3} z_{1}\right)^{3} /\left(z_{1}-z_{2}\right)^{2}\left(z_{2}-z_{3}\right)^{2}\left(z_{1}-z_{3}\right)^{2} .
$$

If $z_{4} \neq \infty$, compute $j$ by applying the transformation $z \mapsto 1 /\left(z-z_{4}\right)$ to each element of $z$. Let $\bar{z}=j^{\prime}$ denote the image of $z$ in $J_{4}$ (the $j$-line).

In particular, points of $\mathcal{H}^{r d}$ over $j^{\prime} \in J_{4}$ are in orbit for the stabilizer of $\boldsymbol{z}$ in $\mathrm{PSL}_{2}(\mathbb{C})$. To see this action explicitly, it suffices to consider a specific choice of $\boldsymbol{z}$. Our choice is this: $\boldsymbol{z}=\{0, \infty,+1,-1\}$. Then $z_{0}=i$ is a convenient choice of base point for the next calculation. Let $\mathcal{L}=\left(\lambda_{0}, \lambda_{\infty}, \lambda_{+1}, \lambda_{-1}\right)$ be an allowable set of generating paths for $\pi_{1}\left(\mathbb{P}^{1} \backslash \boldsymbol{z}, z_{0}\right)$ (as in $\S 6.2$ ). The subscripts allude to respective points $z^{\prime} \in z$. These continuous, semisimplicial paths satisfy the following.

(6.15a) For $z^{\prime} \in z, \lambda_{z^{\prime}}$ is homotopic to a path of the form $\tau_{z^{\prime}} \delta_{z^{\prime}} \tau_{z^{\prime}}^{-1}$ with $\delta_{z^{\prime}}$ the boundary of a clockwise disk around $z^{\prime}$.

(6.15b) The product $\lambda_{0} \lambda_{\infty} \lambda_{-1} \lambda_{1}$ is homotopic to 1 .

(6.15c) For $z^{\prime} \neq z^{\prime \prime}$ paths $\lambda_{z^{\prime}}$ and $\lambda_{z^{\prime \prime}}$ meet only at their initial and end points $z_{0}$.

Applying $\mu^{-1}$ to these paths takes them to a new set of paths starting and ending at the same base point. Suppose $\varphi: X \rightarrow \mathbb{P}_{z}^{1}$ is a cover with $z$ as branch points. Compute branch cycles $\boldsymbol{g}$ for the cover $\varphi$ relative to the paths $\mathcal{L}$. Let $\mu^{-1}(\mathcal{L})$ be the image paths of $\mathcal{L}$ under $\mu^{-1}$. Then, the cover $\mu \circ \varphi$ has $\boldsymbol{g}$ as branch cycle description relative to $\mu^{-1}(\mathcal{L})$.

Suppose $\boldsymbol{g}^{\prime}$ is a description of the branch cycles of $\mu \circ \varphi$ relative to $\mathcal{L}$. Further suppose $Q \in H_{4}$ satisfies $Q(\boldsymbol{g})=\boldsymbol{g}^{\prime}$ ( $Q$ independent of $\boldsymbol{g}$ ). Then, the covering group for $\overline{\mathcal{H}}^{r d} \rightarrow \mathbb{P}_{j}^{1}$ is a quotient of $H_{4} /\langle Q\rangle$. Here is a description of the paths we desire. Let $C$ be the great circle on the Riemann sphere going through $0, i, \infty$. Then $\lambda_{0}$ is the path going from $i$ toward 0 along $C$, down to a neighborhood of 0 where it goes clockwise on a small circle around 0 , and then back up along $C$ to $i$. Similarly, let $\lambda_{\infty}$ be the path on $C$ going from $i$ toward $\infty$, up to a neighborhood of $\infty$ where it goes clockwise on a small circle around $\infty$, and then back along $C$ to $i$. Now, take $\lambda_{+1}$ along a great circle containing $i$ and +1 , confined to the right hemisphere (as $C$ divides it) around +1 appropriate for (6.15). Finally, let $\lambda_{-1}$ be any path around -1 allowed in (6.15) so $\lambda_{-1}$ emanates from $i$ between $\lambda_{+1}$ and $\lambda_{0}$.

Express this choice of $\mathcal{L}$ as words in $\mu^{-1}(\mathcal{L})$. Here is what you get:

(6.16) $\mu^{-1}(\mathcal{L})=(\mathcal{L}) Q^{\prime} Q_{1}^{-1} Q_{3}$ where $Q^{\prime}=Q_{1} Q_{2} Q_{3} Q_{3} Q_{2} Q_{1}$ has the effect of conjugating $\boldsymbol{g}$ by $g_{1}$.

Since $Q^{\prime}$ is already trivial in $H_{4}$, the action of $H_{4}$ on the Nielsen class determines an action of $\bar{H}_{4}$ on $\mathrm{MQ}(G, \mathbf{C})$.

6.4.3. Precise branch cycles. Prop. 6.2 gives precise branch cycles for the pullback cover of $\overline{\mathcal{H}}^{\text {rd }} \rightarrow \mathbb{P}_{j}^{1}$ to the $\lambda$-line. Consider the elements 
$a_{1,2}, a_{1,3}, a_{1,4}$ from $\S 6.1$. Their images in $\bar{H}_{4}$,

$$
a_{1,2}^{\prime}=\left(q_{1}^{\prime}\right)^{-2}, a_{1,3}^{\prime}=q_{1}^{\prime}\left(q_{2}^{\prime}\right)^{-2}\left(q_{1}^{\prime}\right)^{-1}, a_{1,4}^{\prime}=q_{1}^{\prime} q_{2}^{\prime}\left(q_{1}^{\prime}\right)^{-2}\left(q_{2}^{\prime}\right)^{-1}\left(q_{1}^{\prime}\right)^{-1},
$$

generate the fundamental group $\Gamma(2)$ of the $\lambda$-line minus $\{0,1, \infty\}$. It is easy to list six distinct cosets of $\Gamma(2)$ in $\bar{H}_{4}$. We don't know why the branch cycles fell so neatly from the relations, though we are happy they did. There is only one ingredient left to the proof: The relation between $\mathcal{H}_{O^{\prime}}$ being unirational over $K$ and properties of $\overline{\mathcal{H}}_{O^{\prime}}^{r d}$. Consider the easy direction.

Suppose $\mathcal{H}_{O^{\prime}}$ is unirational over $K$. Then there is a copy of $\mathbb{P}_{t}^{1}$ for some variable $t$ that projects onto $\overline{\mathcal{H}}_{O^{\prime}}^{r d}$. One may take the maps here over $K$. So, by Luroth's theorem, $\overline{\mathcal{H}}_{O^{\prime}}^{r d}$ has genus 0 . Being the image of $\mathbb{P}_{t}^{1}$ it contains many $K$ points. These are $K$ divisors of degree 1 . Now, consider the converse.

Suppose $\overline{\mathcal{H}}_{O^{\prime}}^{r d}$ has genus 0 and a $K$ divisor of odd degree. The argument of $\S 2.2$ shows $\overline{\mathcal{H}}_{O^{\prime}}^{r d}$ is isomorphic to $\mathbb{P}_{u}^{1}$ for some function $u$. Choose $u$ to be a generic point on $\overline{\mathcal{H}}_{O^{\prime}}^{r d}$ and let $V_{u}$ be the fiber of $\mathcal{H}_{O^{\prime}} \rightarrow \overline{\mathcal{H}}_{O^{\prime}}^{r d}$ over $u$. Then, $V_{u}$ is an open subset of a variety $V^{\prime}$ isomorphic to $\mathbb{P}^{3}$ over the algebraic closure of $K(u)$. Further, the argument of $\S 6.5$ shows, because $r=4, V_{u}$ has a $K(u)$ rational point. Thus, $V^{\prime}$ is a trivial dimension three Brauer-Severi variety. So, it is birational to $\mathbb{P}^{3}$ over $K(u)$, and $\mathcal{H}_{O^{\prime}}$ is unirational. This concludes the proof of Prop. 6.5.

6.5. Comments on sections to $\mathcal{H} \rightarrow \mathcal{H}^{\text {rd }}$. These comments apply to the spaces $\mathcal{H}=\mathcal{H}^{\text {abs }}(G, \mathbf{C})$ or to $\mathcal{H}^{\text {in }}(G, \mathbf{C})$. Consider that $\mathcal{H}$ is just the fiber product $\mathcal{H}^{r d} \times_{J_{r}} U_{r}$ in $\S 6.1$ notion. The key question that arises in applications is this.

Question 6.7. Suppose for some field $K, \overline{\boldsymbol{p}} \in \mathcal{H}(K)^{r d}$. Then, under what conditions will there exist $\boldsymbol{p} \in \mathcal{H}(K)$ lying over $\overline{\boldsymbol{p}}$ ?

The fiber product formulation is helpful. Suppose $\overline{\boldsymbol{p}}$ lies over $\overline{\boldsymbol{z}} \in J_{r}(K)$. Then, the answer to the question is this:

(6.17) $\boldsymbol{p} \in \mathcal{H}(K)$ over $\overline{\boldsymbol{p}}$ exists if and only if there exists $\boldsymbol{z} \in U_{r}(K)$ lying over $\bar{z}$.

To see this just take $\boldsymbol{p}=(\overline{\boldsymbol{p}}, \boldsymbol{z})$ in the fiber product. When $r=4$ the answer to Question 6.7 is always (at least in characteristic 0).

Proposition 6.8 ( $r=4$-Section). Suppose $j_{0} \in K$. Then, there exists $\boldsymbol{z}$ over $K$ with $j$-invariant equal to $j_{0}$.

Proof. From [Rob73, I.50], $j_{0}=g_{2}^{3} /\left(g_{2}^{3}-27 g_{3}^{2}\right)$ with $Y^{2} Z=4 X^{3}-g_{2} X Z^{2}-$ $g_{3} Z^{3}=0$ a homogeneous equation for an elliptic curve having $j_{0}$ as invariant. So, take $g_{2}=g_{3}=27 j_{0} /\left(j_{0}-1\right)$ to get a curve over $K$ with this invariant. The $K$ point of $\mathbb{P}^{4} \backslash D_{4}$ is the unordered collection $z$ of branch points for this Weierstrass model. 
For all values of $r$, there are applications of the existence of such sections as in Prop. 6.8. For example, Serre has noted the analog for larger values of $r$ does not hold (see [Fri99]).

\section{Appendix on Abhyankar's Lemma.}

Here is a convenient form of Abhyankar's Lemma for its use in $\S 2.5$ and $\S 4.4$.

Lemma 7.1 (Fiber Product Lemma). Let $\psi_{X}: X \rightarrow Z$ and $\psi_{Y}: Y \rightarrow$ $Z$ be nonsingular projective curve covers tamely ramifying over $z_{0} \in Z$. Further, let $\left(x_{1}, \ldots, x_{s}\right)$ be all points of $X$ ramified over $z_{0}$ with respective ramification indices $\left(e_{1}, \ldots, e_{s}\right)$. Similarly, $\left(y_{1}, \ldots, y_{t}\right)$ are all points of $Y$ ramified over $z_{0}$ with respective ramification indices $\left(f_{1}, \ldots, f_{t}\right)$. Let $W$ be the normalization of $X \times_{Z} Y ; \psi_{X}^{*}: W \rightarrow X$ is the natural projection onto the first factor. For each pair $\left(x_{i}, y_{j}\right)$ there are $\operatorname{gcd}\left(e_{i}, f_{j}\right)$ points of $W$ above $x_{i}$, each with ramification index $f_{j} / \operatorname{gcd}\left(e_{i}, f_{j}\right)$. For fixed $i$ as $j$ runs from 1 to $t$, this completely lists points of $W$ over $x_{i}$.

Proof. Everything is local. So the general case reduces to this: $\psi_{X}: X \rightarrow Z$ is $\mathbb{P}_{x}^{1} \rightarrow \mathbb{P}_{z}^{1}, \psi_{X}$ is the $e$-power map, $\psi_{Y}: Y \rightarrow Z$ is $\mathbb{P}_{y}^{1} \rightarrow \mathbb{P}_{z}^{1}, \psi_{Y}$ is the $f$ power map and $z_{0}=0$. Thus, $W$ is $\left\{(x, y) \mid x^{e}-y^{f}=0\right\}$ normalized around $(0,0)$. Let $m$ be the gcd and $n$ the lcm of $e$ and $f$. Assume $\bar{K}$ is algebraically closed and, by the tame ramification assumption, of characteristic prime to $e$ and $f$. Let $R$ be $\bar{K}[x, y] /\left(x^{e}-y^{f}\right)$ localized at $(0,0)$. Further, let $\zeta_{m}$ be a primitive $m$ th root of 1 . Denote $\bar{K}[x]$ localized at $x=0$ by $\bar{K}[x]_{0}$.

The remainder explicitly finds the normalization $S$ of $R$ and lists primes of $S$ over $x=0$ in $\bar{K}[x]_{0}$. Actually, $S$ is $\operatorname{gcd}(e, f)$ copies of $\bar{K}[w]_{0}$ as a $\bar{K}[x]_{0}$ algebra by the embedding $x \mapsto w^{n / m}$. Let $e^{\prime}=e / m$ and $f^{\prime}=f / m$. Euclid's algorithm finds integers $a$ and $b$ with $a f^{\prime}+b e^{\prime}=1: a / e^{\prime}+b / f^{\prime}=1 / e^{\prime} f^{\prime}$. Consider

$$
R_{1}=\bar{K}[u, v]_{(0,0)} /\left(u^{m}-v^{m}\right)=\oplus_{j} \bar{K}[u, v]_{(0,0)} /\left(w-\zeta_{m}^{j} u\right) .
$$

The normalization of $R_{1}$ is the direct sum of $m$ rings isomorphic to $\bar{K}[u]_{0}$. This reduces everything to the case $m=1$. For that, embed $R$ in $S=\bar{K}[w]_{0}$ by $x \mapsto w^{f}$ and $y \mapsto w^{e}$, so $x^{a} y^{b}=w$.

\section{References}

[BF82] R. Biggers and M. Fried, Moduli spaces of covers and the Hurwitz monodromy group, Crelles J., 335 (1982), 87-121.

[Deb99] P. Debes, Arithmétique et espaces de modules de revêtements, 1999, Proceedings of the Schinzel Festschrift, Summer 1997.

[DF90a] P. Debes and M. Fried, Arithmetic variation of fibers in families: Hurwitz monodromy criteria for rational points ... , Crelles J., 409 (1990), 106-137. 
[DF90b] _ Rigidity and real residue class fields, Acta Arith., 56 (1990), 13-45.

[Fri74] M.D. Fried, On Hilbert's irreducibility theorem, J. Number Theory, 6 (1974), 128-146.

[Fri77] , Fields of definition of function fields and Hurwitz families and; groups as Galois groups, Communications in Algebra, 5 (1977), 17-82.

[Fri86] _ Applications of the classification of simple groups to monodromy, part II: Davenport and Hilbert-Siegel problems, preprint, (1986), 1-55.

[Fri90] _ Arithmetic of 3 and 4 branch point covers: A bridge provided by noncongruence subgroups of $\mathrm{SL}_{2}(\boldsymbol{z})$, Progress in Math. Birkhauser, 81 (1990), 77-117.

[Fri95a] _ Enhanced review: Serre's topics in Galois theory, Proceedings of the Recent developments in the Inverse Galois Problem conference, Vol. 186, 1995, AMS Cont. Math series, 15-32.

[Fri95b] _ Extension of constants, rigidity, and the Chowla-Zassenhaus conjecture, Finite Fields and their Applications; Carlitz Volume 1 (1995), 326-359.

[Fri95c] _ Modular towers: Generalizing the relation between dihedral groups and modular curves, Proceedings AMS-NSF Summer Conference, Vol. 186, 1995, Cont. Math series, Recent Developments in the Inverse Galois Problem, 111-171.

[Fri96] _ Alternating groups and lifting invariants, Preprint as of 07/01/96 (1996), $1-34$.

[Fri99]_ Separated variables polynomials and moduli spaces, Zakopane Conference on Number Theory, 1999, Proceedings of the Schinzel Festschrift, Summer 1997.

[FM69] M.D. Fried and R. MacRae, On the invariance of chains of fields, Illinois J. Math., 13 (1969), 167-171.

[GT90] R.M. Guralnick and J.G. Thompson, Finite groups of genus 0, J. of Alg., 131 (1990), 303-341.

[Kan80] W.M. Kantor, Linear groups containing a Singer cycle, J. of Alg., 62 (1980), 232-234.

[MM] G. Malle and B.H. Matzat, Inverse Galois theory, Completed as of Fall 1995.

[Mes90] J.F. Mestre, Extensions régulières de $\mathbb{Q}(t)$ de groupe de Galois $\tilde{A}_{n}$, J. of Alg., 131 (1990), 483-495.

[Mül96] P. Müller, Reducibility behavior of polynomials with varying coefficients, Israel J., 94 (1996), 59-91.

[MF80] D. Mumford and J. Fogerty, Geometric invariant theory, 2nd enlarged ed., Ergebnisse def Mathematik, Vol. 34, Springer-Verlag, 1980.

[Neu93] M. Neubauer, On primitive monodromy groups of genus zero and one, I, Comm. Alg., 21(3) (1993), 711-746.

[Rob73] A. Robert, Elliptic curves, Lecture Notes, Vol. 326, Springer Verlag, Heidelberg, 1973.

[Sch82] A. Schinzel, Selected topics on polynomials, no. ISBN \#0-472-08026-1, University of Michigan Press, 1982.

[Ser92] J.-P. Serre, Topics in galois theory, no. ISBN \#0-86720-210-6, Bartlett and Jones Publishers, 1992.

[Vö196] H. Völklein, Groups as Galois groups, Cambridge Studies in Advanced Mathematics, Vol. 53, Camb. U. Press, Camb. England, 1996. 
[Wie64] H. Wielandt, Finite permutation groups, Academic Press, New York-London, 1964.

Received November 17, 1997 and revised March 27, 1998. Support for second author from NSF \#DMS-9622928 and from Alexander von Humboldt Foundation and Institut für Experimentelle Mathematik, July 1996.

Depart. Mathématiques

UNIV. LILlE

59655 Villeneuve D'AscQ CedeX

FRANCE

E-mail address: pde@ccr.jussieu.fr

UNIVERSITY OF CALIFORNIA

IRVINE, CA 92697

E-mail address: mfried@math.uci.edu 